\begin{tabular}{|c|l|}
\hline Title & Geometric singul arities for solutions of single conservation laws \\
\hline Author(s) & Izumiya, S.; Kossioris, G. T. \\
\hline Citation & Hokkaido University Preprint Series in Mathematics, 211, 1-28 \\
\hline Issue Date & 1993-08 \\
\hline DOI & 10.14943/83355 \\
\hline Doc URL & http://hdl.handle.net/2115/68957 \\
\hline Type & bulletin (article) \\
\hline File Information & pre211.pdf \\
\hline
\end{tabular}

Instructions for use 
GEOMETRIC SINGULARITIES

FOR SOLUTIONS OF SINGLE CONSERVATION LAWS

S. Izumiya and G. T. Kossioris

Series $\sharp 211$. August 1993 


\section{HOKKAIDO UNIVERSITY \\ PREPRINT SERIES IN MATHEMATICS}

$\sharp 184$ : T. Hibi, Canonical modules and Cohen-Macaulay types of partially ordered sets, 6 pages. 1993.

$\sharp 185$ : Y. Giga, K. Yama-uchi, On a lower bound for the extinction time of surfaces moved by mean curvature, 16 pages. 1993.

186: Y. Kurokawa, On functional moduli for first order ordinary differential equations, 9 pages. 1993.

$\sharp 187$ : O. Ogurisu, Unitary equivalence between a spin $1 / 2$ charged particle in a two-dimensional magnetic field and a spin $1 / 2$ neutral particle with an anomalous magnetic moment in a two-dimensional electric field, 4 pages. 1993.

$\sharp 188$ : A. Jensen, T. Ozawa, Existence and non-existence results for wave operators for perturbations of the laplacian, 30 pages. 1993.

$\sharp 189$ : T. Nakazi, Multipliers of invariant subspaces in the bidisc, 12 pages. 1993.

$\sharp 190: \quad$ S. Izumiya, G.T. Kossioris, Semi-local classification of geometric singularities for Hamilton-Jacobi equations, 24 pages. 1993.

$\sharp 191$ : N. Hayashi, T. Ozawa, Finite energy solutions of nonlinear Schrödinger equations of derivative type, 21 pages. 1993.

$\sharp 192$ : J. Seade, T. Suwa, A residue formula for the index of a holomorphic flow, 22 pages. 1993.

$\sharp 193$ : H. Kubo, Blow-up of solutions to semilinear wave equations with initial data of slow decay in low space dimensions, 8 pages. 1993.

\#194: F. Hiroshima, Scaling limit of a model of quantum electrodynamics, 52 pages. 1993.

$\sharp 195$ : T. Ozawa, Y. Tsutsumi, Global existence and asymptotic behavior of solutions for the Zakharov equations in three space dimensions, 34 pages. 1993.

$\sharp 196$ : H. Kubo, Asymptotic behaviors of solutions to semilinear wave equations with initial data of slow decay, 25 pages. 1993.

$\sharp 197$ : Y. Giga, Motion of a graph by convexified energy, 32 pages. 1993.

$\sharp 198$ : T. Ozawa, Local decay estimates for Schrödinger operators with long range potentials, 17 pages. 1993.

$\sharp 199$ : A. Arai, N. Tominaga, Quantization of angle-variables, 31 pages. 1993.

$\sharp 200$ : S. Izumiya, Y. Kurokawa, Holonomic systems of Clairaut type, 17 pages. 1993.

$\sharp 201$ : K.-S. Saito, Y. Watatani, Subdiagonal algebras for subfactors, 7 pages. 1993.

$\sharp 202$ : K. Iwata, On Markov properties of Gaussian generalized random fields, 7 pages. 1993.

$\sharp 203$ : A. Arai, Characterization of anticommutativity of self-adjoint operators in connection with Clifford algebra and applications, 13 pages. 1993.

$\sharp 204$ : J. Wierzbicki, An estimation of the depth from an intermediate subfactor, 7 pages. 1993.

$\sharp 205: \quad$ N. Honda, Vanishing theorem for the tempered distributions, 11 pages. 1993.

$\sharp 206$ : T. Hibi, Betti number sequences of simplicial complexes, Cohen-Macaulay types and Möbius functions of partially ordered sets, and related topics, 25 pages. 1993.

$\sharp 207$ : A. Inoue, Regularly varying correlations, 23 pages. 1993.

$\sharp 208$ : S. Izumiya, B. Li, Overdetermined systems of first order partial differential equations with singular solution, 9 pages. 1993.

\# 209: T. Hibi, Hochster's formula on Betti numbers and Buchsbaum complexes, 7 pages. 1993.

$\sharp 210$ : T. Hibi, Star-shaped complexes and Ehrhart polynomials, 5 pages. 1993. 


\title{
GEOMETRIC SINGULARITIES FOR SOLUTIONS OF SINGLE CONSERVATION LAWS*
}

\author{
SHYUICHI IZUMIYA ${ }^{1}$ AND GEORGIOS T. KOSSIORIS ${ }^{2}$
}

\section{INTRODUCTION}

In this paper we describe the geometric framework for the study of generation and propagation of shock waves in $\mathbb{R}^{n}$ appearing in weak solutions of single conservation laws

$$
\left\{\begin{array}{l}
\frac{\partial y}{\partial t}+\sum_{i=1}^{n} \frac{\partial f_{i}(y)}{\partial x_{i}}=0 \\
y\left(0, x_{1}, \cdots, x_{n}\right)=\phi\left(x_{1}, \cdots, x_{n}\right),
\end{array}\right.
$$

where $f_{i}$ 's and $\phi$ are $C^{\infty}$-functions. Single conservation laws play an important role in various fields, e.g., gas dynamics (see e.g., [12]) and oil reservoir problems (see, e.g., [5]).

The geometric solution $y$ of $(\mathrm{P})$ is defined in the framework of one-parameter unfoldings of immersions and it is constructed by the method of characteristics. Although $y$ is initially smooth there is, in general, a critical time beyond which characteristics cross. The geometric solution past the critical time is multi-valued, that is singularities appear.

The notion of entropy solutions (see [9]) has provided the right weak setting for the study of $(\mathrm{P})$. Existence and uniqueness of the entropy solution of $(\mathrm{P})$ have been established in [9]. The single-valued entropy solution is in general discontinuous and coincides with the smooth geometric solution until the first critical time. After the characteristics cross, the entropy solution develops shock waves, i.e., surfaces across which the entropy solution is discontinuous.

The entropy solution in a neighborhood of the critical time when characteristics cross for the first time has been constructed by Nakane in [11] in the case of $\mathbb{R}^{n}$. The entropy solution is constructed by selecting the proper discontinuous branch of the geometric solution so that the entropy condition is satisfied across the discontinuity. The case $n=1$ has been studied e.g., by Ballou [2], Chen [3], Guckenheimer [7] and Jennings [8].

The framework introduced in [11] describes only the way that shocks are generated. In this paper, we present the geometric framework to study the global

*This work was partially supported by the Army Research Office and the National Science Foundation through the Center for Nonlinear Analysis and by the Japan Association for Mathematical Science

${ }^{1}$ Department of Mathematics, Faculty of Science, Hokkaido University, Sapporo 060, Japan

${ }^{2}$ Department of Mathematics, Carnegie-Mellon University, Pittsburgh, Pennsylvania 15213, USA 
evolution of shock waves. The entropy solution just after the first critical time is a discontinuous single-valued branch of the geometric solution. In order to study the evolution of the shock surface we need to study how the different branches defining the entropy solution evolve in time. As the two branches evolve, the appearance of singularities in a branch affects the form of the discontinuity. Whenever a new singularity appears a new single-valued discontinuous branch has to be chosen so that the entropy condition is satisfied across the discontinuity.

In the present paper, we classify the bifurcations of singularities appearing in a single branch of the multi-valued geometric solution in Sections 3,4. This classification predicts the generic way that shocks are generated and how two shocks interact at their end points. In order to further study the evolution of a shock surface we also classify how various singularities appearing in different branches bifurcate in time in Sections 5,6. This classification describes how one shock interacts with another at a middle point, how different branches evolve from an initially smooth shock surface and how more than two shocks interact.

Acknowledgment. The authors wish to thank the Director of the Center for Nonlinear Analysis M. Gurtin for his support. They would also like to thank C. Dafermos for his constant encouragement regarding this project.

\section{GEOMETRY OF FIRST ORDER QUASI-LINEAR PARTIAL DIFFERENTIAL EQUATIONS}

Since single conservation laws are the special case of first order quasi-linear partial differential equations, we start to study the geometric framework of these equations. We consider a first order quasi-linear partial differential equation:

$$
\sum_{i=1}^{n} a_{i}(x, y) \frac{\partial y}{\partial x_{i}}-b(x, y)=0
$$

where $a_{i}(x, y)(i=1, \ldots, n)$ and $b(x, y)$ are smooth functions. We construct the geometric framework of the above equations in the projective cotangent bundle $\pi: P T^{*}\left(\mathbb{R}^{n} \times \mathbb{R}\right) \rightarrow \mathbb{R}^{n} \times \mathbb{R}$. We now review geometric properties of this space. We consider the tangent bundle $\tau: T P T^{*}\left(\mathbb{R}^{n} \times \mathbb{R}\right) \rightarrow P T^{*}\left(\mathbb{R}^{n} \times \mathbb{R}\right)$ and the differential $\operatorname{map} d \pi: T P T^{*}\left(\mathbb{R}^{n} \times \mathbb{R}\right) \rightarrow T\left(\mathbb{R}^{n} \times \mathbb{R}\right)$ of $\pi$.

For any $X \in T P T^{*}\left(\mathbb{R}^{n} \times \mathbb{R}\right)$, there exists an element $\alpha \in T_{(x, y)}^{*}\left(\mathbb{R}^{n} \times \mathbb{R}\right)$ such that $\tau(X)=[\alpha]$. For an element $V \in T_{(x, y)}\left(\mathbb{R}^{n} \times \mathbb{R}\right)$, the property $\alpha(V)=0$ does not depend on the choice of representative of the class $[\alpha]$. Thus we can define the canonical contact structure on $P T^{*}\left(\mathbb{R}^{n} \times \mathbb{R}\right)$ by

$$
K=\left\{X \in T P T^{*}\left(\mathbb{R}^{n} \times \mathbb{R}\right) \mid \tau(X)(d \pi(X))=0\right\} .
$$

Because of the trivialization $P T^{*}\left(\mathbb{R}^{n} \times \mathbb{R}\right) \cong\left(\mathbb{R}^{n} \times \mathbb{R}\right) \times P\left(\mathbb{R}^{n} \times \mathbb{R}\right)^{*}$, we can choose $\left(\left(x_{1}, \ldots, x_{n}, y\right),\left[\xi_{1} ; \ldots ; \xi_{n} ; \eta\right]\right)$ as the homogeneous coordinate system, where $\left[\xi_{1} ; \ldots ; \xi_{n} ; \eta\right]$ is the homogeneous coordinate of the projective space $P\left(\mathbb{R}^{n} \times\right.$ $\mathbb{R})^{*}$.

It is easy to show that $X \in K_{(x, y),[\xi ; \eta])}$ if and only if $\sum_{i=1}^{n} \mu_{i} \xi_{i}+\lambda \eta=0$, where $d \tilde{\pi}(X)=\sum_{i=1}^{n} \mu_{i} \frac{\partial}{\partial x_{i}}+\lambda \frac{\partial}{\partial y}$. An immersion $i: L \rightarrow P T^{*}\left(\mathbb{R}^{n} \times \mathbb{R}\right)$ is said to be $a$ Legendrian immersion if $\operatorname{dim} L=n$ and $d i_{q}\left(T_{q} L\right) \subset K_{i(q)}$ for any $q \in L$. 
A frst order quasi-linear differential equation (briefly, an equation) is defined to be a hypersurface

$$
E\left(a_{1}, \ldots, a_{n}, b\right)=\left\{((x, y),[\xi ; \eta]) \in P T^{*}\left(\mathbb{R}^{n} \times \mathbb{R}\right) \mid \sum_{i=1}^{n} a_{i}(x, y) \xi_{i}+b(x, y) \eta=0\right\}
$$

A geometric solution of $E\left(a_{1}, \ldots, a_{n}, b\right)$ is a Legendrian submanifold $L$ of $P T^{*}\left(\mathbb{R}^{n} \times\right.$ $\mathbb{R})$ lying in $E\left(a_{1}, \ldots, a_{n}, b\right)$ such that $\pi \mid L$ is an embedding. We consider the meaning of the notion of geometric solutions. Let $S$ be a smooth hypersurface in $\mathbb{R}^{n} \times \mathbb{R}$, then we have a unique Legendrian submanifold $\hat{S}$ in $P T^{*}\left(\mathbb{R}^{n} \times \mathbb{R}\right)$ such that $\pi(\hat{S})=S$ which is given as follows :

$\hat{S}=\left\{((x, y),[\xi ; \eta]) \mid\right.$ the vector $\sum_{i=1}^{n} \xi_{i} \frac{\partial}{\partial x_{i}}+\eta \frac{\partial}{\partial y}$ is a normal vector of $S$ in $\left.\mathbb{R}^{n} \times \mathbb{R}\right\}$.

It follows that if $L$ is a geometric solution of $E\left(a_{1}, \ldots, a_{n}, b\right)$, then we have $L=\widehat{\pi(L)}$.

We consider the condition of a smooth hypersurface $S$ that $\hat{S}$ is contained in $E\left(a_{1}, \ldots, a_{n}, b\right)$. For any $\left(x_{0}, y_{0}\right) \in S$, there exists a smooth submersion germ $f$ : $\left(\mathbb{R}^{n} \times \mathbb{R},\left(x_{0}, y_{0}\right)\right) \rightarrow(\mathbb{R}, 0)$ such that $\left(f^{-1}(0),\left(x_{0}, y_{0}\right)\right)=\left(S,\left(x_{0}, y_{0}\right)\right)$. A vector $\sum_{i=1}^{n} \mu_{i} \frac{\partial}{\partial x_{i}}+\lambda \frac{\partial}{\partial y}$ is tangent to $S$ at $(x, y) \in\left(S,\left(x_{0}, y_{0}\right)\right)$ if and only if it satisfies $\sum_{i=1}^{n} \mu_{i} \frac{\partial f}{\partial x_{i}}+\lambda \frac{\partial f}{\partial y}=0$ at $(x, y)$. Then we have the following representation of $\hat{S}$ :

$$
\left(\hat{S},\left(\left(x_{0}, y_{0}\right) ;\left[\xi_{0} ; \eta_{0}\right]\right)\right)=\left\{\left((x, y),\left[\frac{\partial f}{\partial x} ; \frac{\partial f}{\partial y}\right]\right) \mid(x, y) \in\left(S,\left(x_{0}, y_{0}\right)\right)\right\}
$$

By the classical theory of first order partial differential equations, the characteristic vector field of $E\left(a_{1}, \ldots, a_{n}, b\right)$ is defined to be

$$
X\left(a_{1}, \ldots, a_{n}, b\right)=\sum_{i=1}^{n} a_{i}(x, y) \frac{\partial}{\partial x_{i}}+b(x, y) \frac{\partial}{\partial y}
$$

Then we have the following characterization theorem of geometric solutions.

Proposition 1.1. Let $S$ be a smooth hypersurface in $\mathbb{R}^{n} \times \mathbb{R}$. Then $\hat{S}$ is a geometric solution of $E\left(a_{1}, \ldots, a_{n}, b\right)$ if and only if the characteristic vector field $X\left(a_{1}, \ldots, a_{n}\right.$, $b)$ is tangent to $S$.

Proof. We have a local representation

$$
\left(\hat{S},\left(\left(x_{0}, y_{0}\right) ;\left[\xi_{0} ; \eta_{0}\right]\right)\right)=\left\{\left((x, y),\left[\frac{\partial f}{\partial x} ; \frac{\partial f}{\partial y}\right]\right) \mid(x, y) \in\left(S,\left(x_{0}, y_{0}\right)\right)\right\}
$$

where $f:\left(\mathbb{R}^{n} \times \mathbb{R},\left(x_{0}, y_{0}\right)\right) \rightarrow(\mathbb{R}, 0)$ is a submersion germ with $f^{-1}(0)=S$. It follows that $\hat{S} \subset E\left(a_{1}, \ldots, a_{n}, b\right)$ if and only if

$$
\sum_{i=1}^{n} a_{i}(x, y) \frac{\partial f}{\partial x_{i}}+b(x, y) \frac{\partial f}{\partial y}=0
$$


for $(x, y) \in\left(S,\left(x_{0}, y_{0}\right)\right)$. The last condition is equivalent to the condition that $X\left(a_{1}, \ldots, a_{n}, b\right)$ is tangent to $S$ at $(x, y)$.

The Cauchy problem can be solved by the method of characteristic. We say that a geometric Cauchy problem $(G C P)$ is given for an equation $E\left(a_{1}, \ldots, a_{n}, b\right)$ if there is given an (n-1)-dimensional submanifold $i^{\prime}: S^{\prime} \subset \mathbb{R}^{n} \times \mathbb{R}$ such that the characteristic vector field $X\left(a_{1}, \ldots, a_{n}, b\right)$ is not tangent to $S^{\prime}$. The following result is well-known (cf. [1]), however we clarify the geometric situation in this paper.

Theorem 1.2 (Existence theorem). A GCP $i^{\prime}: S^{\prime} \subset \mathbb{R}^{n} \times \mathbb{R}$ has a unique geometric solution, that is there exists a smooth hypersurface $S \subset \mathbb{R}^{n} \times \mathbb{R}, S^{\prime} \subset S$ such that $\hat{S} \subset E\left(a_{1}, \ldots, a_{n}, b\right)$, and any two such smooth hypersurfaces coincide in a neighborhood of $S^{\prime}$.

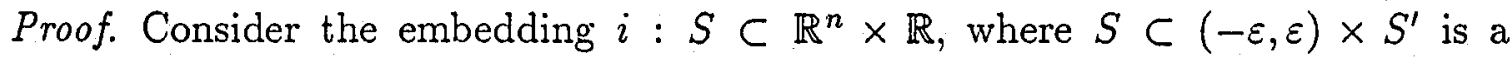
neighborhood of $0 \times S^{\prime}, \varepsilon>0$; here $i(t, q)=T_{t}(q), q \in S^{\prime},(t, q) \in S$, and $T_{t}$ is an one-parameter group of translations along $X\left(a_{1}, \ldots, a_{n}, b\right)$. By the construction, we have $X\left(a_{1}, \ldots, a_{n}, b\right) \in T S$, so that $\hat{S} \subset E\left(a_{1}, \ldots, a_{n}, b\right)$ by Proposition 1.1.

On the other hand, for any geometric solution $L$ of $E\left(a_{1}, \ldots, a_{n}, b\right), \pi(L)$ must be invariant under $X\left(a_{1}, \ldots, a_{n}, b\right)$, so that it is coincide with $S$ in some neighborhood.

We consider the canonical projection $\pi_{1}: \mathbb{R}^{n} \times \mathbb{R} \rightarrow \mathbb{R}^{n}$ given by $\pi_{1}(x, y)=x$. Let $L$ be a geometric solution of $E\left(a_{1}, \ldots, a_{n}, b\right)$. We say that $q \in L$ is a singular point if $\operatorname{rank} d\left(\pi_{1} \circ \pi \mid L\right) \varsubsetneqq n$. We remark that $q \in L$ is not a singular point if and only if there exists a smooth function germ $f:\left(\mathbb{R}^{n}, x_{0}\right) \rightarrow \mathbb{R}$ such that $\left(S,\left(x_{0}, y_{0}\right)\right)=$ (graph $\left.f,\left(x_{0}, y_{0}\right)\right)$, where $x_{0}=\pi_{1} \circ \pi(q), y_{0}=f\left(x_{0}\right)$ and graph $f=\{(x, f(x)) \mid x \in$ $\left.\left(\mathbb{R}^{n}, x_{0}\right)\right\}$.

\section{EVOLUTION EQUATIONS}

From now on we consider the following equations:

$$
\frac{\partial y}{\partial t}+\sum_{i=1}^{n} a_{i}(t, x, y) \frac{\partial y}{\partial x_{i}}-b(t, x, y)=0
$$

where $a_{i}(t, x, y)(i=1, \ldots, n)$ and $b(t, x, y)$ are smooth functions. In this case we can construct the geometric framework in the projective cotangent bundle $\Pi: P T^{*}\left(\mathbb{R} \times \mathbb{R}^{n} \times \mathbb{R}\right) \rightarrow \mathbb{R} \times \mathbb{R}^{n} \times \mathbb{R}$ with the trivialization $P T^{*}\left(\mathbb{R} \times \mathbb{R}^{n} \times\right.$ $\mathbb{R}) \cong\left(\mathbb{R} \times \mathbb{R}^{n} \times \mathbb{R}\right) \times P\left(\mathbb{R} \times \mathbb{R}^{n} \times \mathbb{R}\right)^{*}$. and the natural homogeneous coordinate $\left(\left(t, x_{1}, \ldots, x_{n}, y\right),\left[\sigma ; \xi_{1} ; \ldots ; \xi_{n} ; \eta\right]\right)$. We also adopt the canonical contact structure $K$ on $P T^{*}\left(\mathbb{R} \times \mathbb{R}^{n} \times \mathbb{R}\right)$.

The equation is a hypersurface

$$
\begin{aligned}
E\left(1, a_{1}, \ldots, a_{n}, b\right)=\{((t, x, y),[\sigma ; \xi ; \eta]) & \in P T^{*}\left(\mathbb{R} \times \mathbb{R}^{n} \times \mathbb{R}\right) \mid \\
\sigma & \left.+\sum_{i=1}^{n} a_{i}(t, x, y) \xi_{i}+b(t, x, y) \eta=0\right\}
\end{aligned}
$$

and the characteristic vector field is given by

$$
X\left(1, a_{1}, \ldots, a_{n}, b\right)=\frac{\partial}{\partial t}+\sum_{i=1}^{n} a_{i}(t, x, y) \frac{\partial}{\partial x_{i}}+b(t, x, y) \frac{\partial}{\partial y}
$$


We remark that the dimension of geometric solutions is $n+1$.

We usually distinguish the time and the space, however GCP is not enough to serve to consider this case. We need a more restricted framework.

We say that a geometric Cauchy problem associated with the time parameter (GCPT) is given for an equation $E\left(1, a_{1}, \ldots, a_{n}, b\right)$ if a GCP $i^{\prime}: S^{\prime} \subset \mathbb{R} \times \mathbb{R}^{n} \times \mathbb{R}$ with $i^{\prime}\left(S^{\prime}\right) \subset c \times \mathbb{R}^{n} \times \mathbb{R}$ for some $c \in \mathbb{R}$ is given. Since $X\left(1, a_{1}, \ldots, a_{n}, b\right) \notin$ $T\left(c \times \mathbb{R}^{n} \times \mathbb{R}\right)$, the condition $X\left(1, a_{1}, \ldots, a_{n}, b\right) \notin T S^{\prime}$ is automatically satisfied when $i^{\prime}\left(S^{\prime}\right) \subset c \times \mathbb{R}^{n} \times \mathbb{R}$. It follows that GCPT is given if and only if there exists an $n$-dimensional submanifold $i^{\prime}: S^{\prime} \subset c \times \mathbb{R}^{n} \times \mathbb{R}$ for some $c \in \mathbb{R}^{n}$.

Remark. The Cauchy problem $y(0, x)=\phi(x)$ is a GCPT. The initial submanifold is given by

$$
S_{\phi, 0}=\left\{(0, x, \phi(x)) \mid x \in \mathbb{R}^{n}\right\} .
$$

Let $i: S^{\prime} \subset 0 \times \mathbb{R}^{n} \times \mathbb{R}$ be an initial submanifold of GCPT. By. Theorem 1.2, we can construct a unique geometric solution $\hat{S}$ such that $S \mid 0 \times \mathbb{R}^{n} \times \mathbb{R}=S^{\prime}$. Since $X\left(1, a_{1}, \ldots, a_{n}, b\right) \in T S, S$ is transverse to $c \times \mathbb{R}^{n} \times \mathbb{R}$ for any $c \in(\mathbb{R}, 0)$. It follows that $S_{c}=S \cap\left(c \times \mathbb{R}^{n} \times \mathbb{R}\right)$ is also an $n$-dimensional submanifold. The problem of studying the singularities of the geometric solution is formulated as follows:

Geometric problem. Classify the generic bifurcations of singularities of the oneparameter family of smooth mappings

$$
\tilde{\pi}_{t} \mid: S_{t} \rightarrow \mathbb{R}^{n}
$$

with respect to the time parameter $t$, where $\tilde{\pi}_{t}: t \times \mathbb{R}^{n} \times \mathbb{R} \rightarrow \mathbb{R}^{n}$ is given by $\tilde{\pi}(t, x, y)=x$.

In order to study the singularities of the geometric solution we identify geometric solutions with one-parameter unfoldings of immersions. Such a characterization, which is given in Section 4 permits the use of the available singularity theory of oneparameter unfoldings of immersions. In the next section we present the necessary background material that we use in Section 4.

\section{ONE PARAMETER UNFOLDINGS OF IMMERSIONS}

We now describe the notion of unfoldings of immersions. Let $R$ be an $(n+1)$ dimensional smooth manifold and $\mu\left(R, u_{0}\right) \rightarrow\left(\mathbb{R}, t_{0}\right)$ be a submersion germ. We call a map germ $\mathcal{I}:\left(R, u_{0}\right) \rightarrow \mathbb{R} \times \mathbb{R}^{n} \times \mathbb{R}$ of the form $\mathcal{I}(t, x)=(\mu(u), x(u), y(u))$ an unfolding of immersions if $\mathcal{I}$ is an immersion germ and $\mathcal{I} \mid \mu^{-1}(t)$ is also an immersion for each $t \in\left(\mathbb{R}, t_{0}\right)$.

In order to study bifurcations of singularities of unfoldings of immersions, we introduce the following equivalence relation. Let $\mathcal{I}_{i}:\left(R, u_{i}\right) \rightarrow\left(\mathbb{R} \times \mathbb{R}{ }^{n} \times \mathbb{R},\left(t_{i}, x_{i}, y_{i}\right)\right.$ $(i=0,1)$ be unfoldings of immersions. We say that $\mathcal{I}_{0}$ and $\mathcal{I}_{1}$ are $t$-P-A-equivalent if there exist a diffeomorphism germ

$$
\Phi:\left(\mathbb{R} \times\left(\mathbb{R}^{n} \times \mathbb{R}\right),\left(t_{0}, x_{0}, y_{0}\right)\right) \rightarrow\left(\mathbb{R} \times\left(\mathbb{R}^{n} \times \mathbb{R}\right),\left(t_{1}, x_{1}, y_{1}\right)\right)
$$

of the form

$$
\Phi(t, x, y)=\left(\phi_{1}(t), \phi_{2}(t, x), \phi_{3}(t, x, y)\right)
$$


and a diffeomorphism germ $\Psi:\left(R, u_{0}\right) \rightarrow\left(R, u_{1}\right)$ such that $\hat{\Phi} \circ \mathcal{I}_{0}=\mathcal{I}_{1} \circ \Psi$.

In an analogous way to that of Arnol'd-Zakalyukin's theory for Legendrian singularities ([15]), we can construct generating families of unfoldings of immersions. Let $F:\left(\mathbb{R} \times \mathbb{R}^{n} \times \mathbb{R}, 0\right) \rightarrow(\mathbb{R}, 0)$ be a function germ such that $F \mid\{0\} \times \mathbb{R}^{n} \times \mathbb{R}$ is submersive. Then $F^{-1}(0)$ is a smooth $(n+1)$-manifold and $\pi_{F}:\left(F^{-1}(0), 0\right) \rightarrow \mathbb{R}$ is a submersion, where $\pi(t, x, y)=t$. Let $\mathcal{I}:\left(R, u_{0}\right) \rightarrow\left(\mathbb{R} \times \mathbb{R}^{n} \times \mathbb{R},\right)$ be an unfolding of immersions. Since the image of $\mathcal{I}$ is a codimension one submanifold germ of $\mathbb{R} \times \mathbb{R}^{n} \times \mathbb{R}$ such that each Image $\mathcal{I}\left(\mu^{-1}(t)\right)$ is also a codimension one submanifold of $\{t\} \times \mathbb{R}^{n} \times \mathbb{R}$, We can choose a function germ $\tilde{F}:\left(\mathbb{R} \times \mathbb{R}^{n} \times \mathbb{R},\left(t_{0}, x_{0}, y_{0}\right)\right) \rightarrow(\mathbb{R}, 0)$ such that $F \mid\left\{t_{0}\right\} \times \mathbb{R}^{n} \times \mathbb{R}$ is submersive and $F^{-1}(0)=$ Image $\mathcal{I}$. We define a function germ $F:\left(\mathbb{R} \times \mathbb{R}^{n} \times \mathbb{R},(0)\right) \rightarrow(\mathbb{R}, 0)$ by $F(t, x, y)=\tilde{F}\left(t+t_{0}, x+x_{0}, y+y_{0}\right)$. We call $F$ a generating family of $\mathcal{I}$. We remark that if $F_{i}(i=0,1)$ are generating families of the same unfolding of immersions, then there exists a function germ $\lambda:\left(\mathbb{R} \times \mathbb{R}^{n} \times \mathbb{R}, 0\right) \rightarrow(\mathbb{R}, 0)$ with $\lambda(0) \neq 0$ such that $\lambda(t, x, y) \cdot F_{0}(t, x, y)=F_{1}(t, x, y)$. We also consider an equivalence relation among generating families of regular Legendrian unfoldings. Let

$$
F_{i}:\left(\mathbb{R} \times \mathbb{R}^{n} \times \mathbb{R}, 0\right) \rightarrow(\mathbb{R}, 0)
$$

be generating families of unfoldings of immersions $\mathcal{I}_{i}(i=0,1)$. We say that $F_{0}$ and $F_{1}$ are $t-P-\mathcal{K}$-equivalent if there exists a diffeomorphism germ

$$
\Phi:\left(\mathbb{R} \times \mathbb{R}^{n} \times \mathbb{R}, 0\right) \rightarrow\left(\mathbb{R} \times \mathbb{R}^{n} \times \mathbb{R}, 0\right)
$$

of the form

$$
\Phi(t, x, y)=\left(\phi_{1}(t), \phi_{2}(t, x), \phi_{3}(t, x, y)\right)
$$

such that

$$
\left\langle F_{1} \circ \Phi\right\rangle_{\mathcal{E}_{(t, x, y)}}=\left\langle F_{0}\right\rangle_{\mathcal{E}_{(t, x, y)}}
$$

where $\left\langle F_{0}\right\rangle_{\mathcal{E}_{(t, x, y)}}$ denotes the ideal generated by $F_{0}$ in the ring $\mathcal{E}_{(t, x, y)}$ of function germs of $(t, x, y)$-variables at the origin.

For a generating family $F$ of $\mathcal{I}$, we define

$$
T_{e}(P-\mathcal{K})(f)=\left\langle\frac{\partial f}{\partial y}, f\right\rangle_{\mathcal{E}_{(x, y)}}+\left\langle\frac{\partial f}{\partial x_{1}}, \ldots, \frac{\partial f}{\partial x_{n}}\right\rangle_{\mathcal{E}_{x}}
$$

and $P-\mathcal{K}$-cod $f=\operatorname{dim}_{\mathbb{R}} \mathcal{E}_{(x, y)} / T_{e}(\mathrm{P}-\mathcal{K})(f)$, where $f=F \mid 0 \times \mathbb{R}^{n} \times \mathbb{R}$. We also say that $F$ is a P-K-versal deformation of $f$ if

$$
\mathcal{E}_{(x, y)}=\left\langle\frac{\partial F}{\partial t} \mid 0 \times \mathbb{R}^{n} \times \mathbb{R}\right\rangle_{\mathbb{R}}+T_{e}(\mathrm{P}-\mathcal{K})(f) .
$$

By definition, we have the following simple proposition.

Proposition 3.2. Let $F_{i}(i=0,1)$ be generating families of unfoldings of immersions $\mathcal{I}_{i}$. Then $\mathcal{I}_{0}$ and $\mathcal{I}_{1}$ are $t-P$-A-equivalent if and only if $F_{0}$ and $F_{1}$ are t-P-K-equivalent.

We can classify generic unfoldings of immersions under the $t$ - $P$ - $\mathcal{A}$-equivalence for any fixed $n$. In [15], Zakalyukin has given a generic classification of function germs $F:\left(\mathbb{R} \times \mathbb{R}^{n} \times \mathbb{R}^{k}, 0\right) \rightarrow(\mathbb{R}, 0)$ under the $t$ - $P$ - $\mathcal{K}$-equivalence for $n \leq 5$. In our case, $k=1$, so that we can detect generic normal form for any $n$ by the same method as that of in Zakalyukin ([15], Part 2.2). Since the set of function germs $F(t, x, y)$ with submersive function $F \mid\{0\} \times \mathbb{R}^{n} \times \mathbb{R}$ is open, we have the following theorem as a corollary of Zakalyukin's theorem. 
Theorem 3.3. For the generic unfolding of immersions $\mathcal{I}$, the generating family $F$ is $t-P$ - $\mathcal{K}$-equivalent to one of the germs in the following list:

$$
y^{k+1}+\sum_{i=1}^{k} x_{i} y^{i-1}(1 \leq k \leq n)
$$

$$
y^{k+1}+y^{k-1}\left(t \pm x_{k}^{2} \pm \cdots \pm x_{n}^{2}\right)+\sum_{i=1}^{k-1} x_{i} y^{i-1}(2 \leq k \leq n+1)
$$

Remarks. Each germ $F$ in the above theorem is a P-K-versal deformation of $f=\left.F\right|_{\{0\} \times \mathbb{R}^{n} \times \mathbb{R} \text {. }}$

On the other hand, we can prove that $F$ is "structurally stable" relative to the $t$ - $P$ - $\mathcal{K}$-equivalence if and only if $F$ is a $P$ - $\mathcal{K}$-versal deformation of $f$ by using the ordinary method of singularity theory (cf. $[6,10,13])$. By this fact, we can assert that the above theorem gives a classification of structural stable one-parameter unfoldings of immersions, so that we call the corresponding unfolding of immersions in the above list a stable unfolding of immersions. We argue the stability of these in Appendix A. We only give the list of these unfoldings of immersions:

$$
\left(t, u_{n}^{k+1}+\sum_{i=1}^{k-1} u_{i} u_{n}^{i}, u_{1}, \ldots, u_{n}\right)(1 \leq k \leq n)
$$

$\left({ }^{1} A_{k}\right)\left(t, u_{n}^{k+1}+u_{n}^{k-1}\left(t \pm u_{k-1}^{2} \pm \cdots \pm u_{n-1}^{2}\right)+\sum_{i=1}^{k} u_{i} u_{n}^{i}, u_{1}, \ldots, u_{n}\right)(2 \leq k \leq n+1)$

We also remark that the germ of type ${ }^{1} A_{2}$ describes the situation that how the singularity of the geometric solution appears or vanishes.

\section{REAlization theOREMS}

In this section we identify the geometric solution of a (GCPT) introduced in Section 2 with the notion of unfoldings of immersions.

Let $i: S^{\prime} \subset 0 \times \mathbb{R}^{n} \times \mathbb{R}$ be an initial submanifold of GCPT. By Theorem 1.2, we can construct a unique geometric solution $\hat{S}$ such that $S \mid 0 \times \mathbb{R}^{n} \times \mathbb{R}=S^{\prime}$. Since $X\left(1, a_{1}, \ldots, a_{n}, b\right) \in T S, S$ is transverse to $c \times \mathbb{R}^{n} \times \mathbb{R}$ for any $c \in(\mathbb{R}, 0)$. It follows that $S_{c}=S \cap\left(c \times \mathbb{R}^{n} \times \mathbb{R}\right)$ is also an $n$-dimensional submanifold. If we consider the local parametrization of $S$, we may assume that $S$ is the image of an immersion germ $\mathcal{I}:\left(\mathbb{R} \times \mathbb{R}^{n}, 0\right) \rightarrow \mathbb{R} \times \mathbb{R}^{n} \times \mathbb{R}$ such that $\mathcal{I} \mid c \times \mathbb{R}^{n}$ is also an immersion for any $c \in(\mathbb{R}, 0)$. Hence we can choose the coordinate representation of $\mathcal{I}$ as $\mathcal{I}(t, x)=(t, x(t, u), y(t, u))$. This completes the proof of first part of Theorem 4.1 . 
Theorem 4.1. (1) The local geometric solution of the geometric Cauchy problem associated with the time parameter for the quasi-linear first order partial differential equation $E\left(1, a_{1}, \ldots, a_{n}, b\right)$ is Image $\mathcal{I}$, where $\mathcal{I}:\left(\mathbb{R} \times \mathbb{R}^{n}, 0\right) \rightarrow \mathbb{R} \times \mathbb{R}^{n} \times \mathbb{R}$ is an unfolding of immersions.

(2) Let $\mathcal{I}:\left(\mathbb{R} \times \mathbb{R}^{n}, 0\right) \rightarrow \mathbb{R} \times \mathbb{R}^{n} \times \mathbb{R}$ be an unfolding of immersions. Then there exist function germs $a_{i}(t, x, y)(i=1, \ldots, n$ and $b(t, x, y)$ such that Image $\mathcal{I}$ is a local geometric solution of the geometric Cauchy problem associated with the time parameter for the quasi-linear first order partial differential equation $E\left(1, a_{1}, \ldots, a_{n}, b\right)$, where the initial submanifold is $\mathcal{I}\left(0 \times \mathbb{R}^{n}\right)$.

Proof. The assertion (1) is already proved by the previous arguments. We prove the assertion (2). By the definition, we have $\mathcal{I}(t, x)=(t, x(t, u), y(t, u))$. Since $\mathcal{I}$ is an immersion germ, we have an epimorphism

$$
\mathcal{I}^{*}: C_{\mathcal{I}(0)}^{\infty}\left(\mathbb{R} \times \mathbb{R}^{n} \times \mathbb{R}\right) \rightarrow C_{0}^{\infty}\left(\mathbb{R} \times \mathbb{R}^{n}\right),
$$

defined by $\mathcal{I}^{*}(h)=h \circ \mathcal{I}$, where $\mathcal{I}^{*}: C_{\mathcal{I}(0)}^{\infty}\left(\mathbb{R} \times \mathbb{R}^{n} \times \mathbb{R}\right)$ is the ring of function germs at $\mathcal{I}(0)$. It follows that there exist $a_{i}, b \in \mathcal{I}^{*}: C_{\mathcal{I}(0)}^{\infty}\left(\mathbb{R} \times \mathbb{R}^{n} \times \mathbb{R}\right)$ such that

$$
a_{i}(t, x(t, u), y(t, u))=\frac{d y}{d t}(t, u) \quad(i=1, \ldots, n)
$$

and

$$
b(t, x(t, u), y(t, u))=\frac{d y}{d t}(t, u)
$$

These relations denote that each curve $\mathcal{I}(t, u)$ from $\mathcal{I}(0, u)$ is a characteristic flows, so that $X\left(1, a_{1}, \ldots, a_{n}, b\right) \in T \mathcal{I}\left(\mathbb{R} \times \mathbb{R}^{n}\right)$. By Proposition 1.1, Image $\mathcal{I}$ is a geometric solution of $E\left(1, a_{1}, \ldots, a_{n}, b\right)$.

Remark. Let $F:\left(\mathbb{R} \times \mathbb{R}^{n} \times \mathbb{R}, 0\right) \rightarrow(\mathbb{R}, 0)$ be a generating family of an unfolding of immersions $\mathcal{I}$. If Image $\mathcal{I}$ is a geometric solution of $E\left(1, a_{1}, \ldots, a_{n}, b\right)$, then we have

$$
\frac{\partial F}{\partial t}+\sum_{i=1}^{n} a_{i}(t, x, y) \frac{\partial F}{\partial x_{i}}+b(t, x, y) \frac{\partial F}{\partial y}=0 \text { on } F^{-1}(0) \text {. }
$$

The above theorem guarantees that the class of unfoldings of immersions supplies the correct class to describe the geometric solutions of (GCPT) for first order quasi linear partial differential equations of evolution type. Thus, generic results for the singularities of unfoldings of immersions can be translated to generic results in the class of all first order quasi linear partial differential equations $E\left(1, a_{1}, \ldots, a_{n}, b\right)$ and all initial conditions. However, our purpose is to find generic normal forms of singularities for geometric solutions of single conservation laws, so that we must also concern ourselves with what are the types of singularities that the geometric solution to a given first order quasi linear partial differential equation might exhibit. Representation Theorems 4.2-4.4 address this question.

Let $i:\left(S^{\prime}, u_{0}\right) \rightarrow \mathbb{R}^{n} \times \mathbb{R}$ be an immersion germ, where $\operatorname{dim} S^{\prime}=n$. We define an immersion germ $\tilde{i}:\left(\mathbb{R} \times S^{\prime},\left(0, u_{0}\right)\right) \rightarrow \mathbb{R} \times \mathbb{R}^{n} \times \mathbb{R}$ by $\tilde{i}(t, u)=(t ; i(u))$. We call $\tilde{i}$ a trivial unfolding of immersions induced by $i$. Let $\mathcal{I}:\left(R, u_{0}\right) \rightarrow \mathbb{R} \times \mathbb{R}^{n} \times \mathbb{R}$ be an unfolding of immersion germs. We say that $\mathcal{I}$ has a trivial bifurcation if $\mathcal{I}$ is $t$-P-A-equivalent to a trivial unfolding of immersions induced by $\mathcal{I} \mid \mu^{-1}\left(t_{0}\right)$. Then we have the following theorem 
Theorem 4.2. Let $E\left(1, a_{1}, \ldots, a_{n} . b\right)$ be a quasi-linear first order partial differential equation and $\mathcal{I}:\left(R, u_{0}\right) \rightarrow \mathbb{R} \times \mathbb{R}^{n} \times \mathbb{R}$ be a stable unfolding of immersions. If $I$ has a trivial bifurcation, then there exists an unfolding of immersions $\mathcal{I}^{\prime}$ such that Image $\mathcal{I}^{\prime}$ is a local geometric solution of $E\left(1, a_{1}, \ldots, a_{n} . b\right)$ and $\mathcal{I}, \mathcal{I}^{\prime}$ are $t-P$ $\mathcal{A}$-equivalent.

Proof. Let $G:\left(\mathbb{R} \times \mathbb{R}^{n} \times \mathbb{R}, 0\right) \rightarrow(\mathbb{R}, 0)$ be a generating family of the unfolding of immersions $\mathcal{I}$. Since $G \mid\{0\} \times \mathbb{R}^{n} \times \mathbb{R}$ is submersive, the set $\phi_{g}=\{(0, x, y) \mid g(x, y)=0\}$ is an initial condition for the (GCPT), where $g=G \mid\{0\} \times \mathbb{R}^{n} \times \mathbb{R}$. By the argument of the proof of the first part of we can construct an unfolding of immersions $\mathcal{I}^{\prime}$ which is a locally unique geometric solution around $\phi_{g}$.

We now choose a generating family $F:\left(\mathbb{R} \times \mathbb{R}^{n} \times \mathbb{R}, 0\right) \rightarrow(\mathbb{R}, 0)$ of $\mathcal{I}^{\prime}$. By definition, $F^{-1}(0) \mid\{0\} \times \mathbb{R}^{n} \times \mathbb{R}=\phi_{g}$, so we may assume that $f$ and $g$ are P$\mathcal{K}$-equivalent, where $f=F \mid\{0\} \times \mathbb{R}^{n} \times \mathbb{R}$. Since $F$ is a $P$-K -versal deformation of $f$ and $\mathcal{I}$ has a trivial bifurcation, $\frac{\partial F}{\partial t} \mid\{0\} \times \mathbb{R}^{n} \times \mathbb{R} \in T_{e}(P-\mathcal{K})(f)$, and hence $\mathcal{E}_{(x, y)}=T_{e}(P-\mathcal{K})(f)$. Therefore, it follows that $\mathcal{E}_{(x, y)}=T_{e}(P-\mathcal{K})(g)$ and $G$ is also a $P$-K -versal deformation of $g$. By the uniqueness theorem of $P$-K $\mathcal{K}$-versal deformations (see [4]), $F$ and $G$ are $t$-P-K-equivalent. This completes the proof.

We remark that ${ }^{0} A_{k}$-type germs in Theorem 3.3 can always be realized as geometric solutions for any first order quasi-linear partial differential equation. However, if an unfolding of immersions has a non-trivial bifurcation, the situation is different as follows:

Example 4.3. Consider the equation: $\frac{\partial y}{\partial t}+y^{2} \frac{\partial y}{\partial x_{i}}=0$.

We now consider the function germ $f(x, y)=y^{3}+x$, then $\phi_{f}=\left\{\left(-y^{3}, y\right) \mid y \in\right.$ $(\mathbb{R}, 0)\}$ is a curve in $(\mathbb{R} \times \mathbb{R}, 0)$. We adopt $\phi_{f}$ as an initial condition for the (GCPT), then we can get an unfolding of immersion $\mathcal{I}$ by the method of characteristics. Let $G(t, x, y)$ be a generating family of $\mathcal{I}$, then $g(x, y)=G(0, x, y)$ satisfies that $\phi_{f}=g^{-1}(0)$. It follows that there exists $\lambda \in \mathcal{E}_{(x, y)}$ such that $\lambda(0) \neq 0$ and $f(x, y)=\lambda(x, y) g(x, y)$. Define a function germ $F:(\mathbb{R} \times \mathbb{R} \times \mathbb{R}, 0) \rightarrow(\mathbb{R}, 0)$ by $F(t, x, y)=\lambda(x, y) G(t, x, y)$, then we have $F^{-1}(0)=G^{1}(0)=$ Image $\mathcal{I}$. and $F(0, x, y)=f(x, y)$. It follows that $\frac{\partial F}{\partial t}+y^{2} \frac{\partial F}{\partial x}=0$ on $F^{-1}(0)$. Then we have $\frac{\partial F}{\partial t}+y^{2} \frac{\partial F}{\partial x} \equiv 0 \bmod \langle F\rangle_{\mathcal{E}_{(t, x, y)}}$. Since $F(0, x, y)=f(0, x, y)$, we have $\left.\frac{\partial F}{\partial t}\right|_{t=0}+y^{2} \in$ $\left\langle y^{3}+x\right\rangle_{\mathcal{E}_{(x, y)}}$. We can easily calculate that $T_{e}(P-\mathcal{K})(f)=\left\langle y^{2}, x\right\rangle_{\left(\mathcal{E}_{(x, y)}\right.}+\mathcal{E}_{x}$, so that $\frac{\partial F}{\partial t} \in T_{e}(P-\mathcal{K})(f)$. This formula show that $F$ cannot be a $P$-K $\mathcal{K}$-versal deformation of $f$. However the generating family of type ${ }^{1} A_{2}: y^{3}+y t+x$ is a $P$ - $\mathcal{K}$-versal deformation of $f$. Then $F$ is not $t$ - $P$ - $\mathcal{K}$-equivalent to germ of type ${ }^{1} A_{2}$. Since $F$ and $G$ are $t-P-\mathcal{K}$-equivalent, $G$ is not $t-P-\mathcal{K}$-equivalent to germ of type ${ }^{1} A_{2}$. Thus the unfolding of immersions of type ${ }^{1} A_{2}$ cannot be realized as a geometric solution of $\frac{\partial y}{\partial t}+y^{2} \frac{\partial y}{\partial x_{i}}=0$.

Hence, we assume a kind of non-degeneracy condition on the first order quasilinear partial differential equation. We say that a first order quasi-linear partial differential equation $E\left(1, a_{1}, \ldots, a_{n}, b\right)$ is $k$-non-degenerated at $\left(t_{0}, x_{0}, y_{0}\right)$ if

$$
\frac{\partial^{k} a}{\partial y^{k}}\left(t_{0}, x_{0}, y_{0}\right) \neq 0
$$


where $\frac{\partial^{k} a}{\partial y^{k}}\left(t_{0}, x_{0}, y_{0}\right)=\left(\frac{\partial^{k} a_{1}}{\partial y^{k}}\left(t_{0}, x_{0}, y_{0}\right), \ldots, \frac{\partial^{k} a_{n}}{\partial y^{k}}\left(t_{0}, x_{0}, y_{0}\right)\right)$. We simply say that $E\left(1, a_{1}, \ldots, a_{n}, b\right)$ is non-degenerated at $\left(t_{0}, x_{0}, y_{0}\right)$ if it is 1 -non-degenerated at $\left(t_{0}, x_{0}, y_{0}\right)$. Then we have the following realization theorem.

Theorem 4.4. Let $E\left(1, a_{1}, \ldots, a_{n}, b\right)$ be a first order quasi-linear partial differential equation and $\mathcal{I}:\left(R, u_{0}\right) \rightarrow \mathbb{R} \times \mathbb{R}^{n} \times \mathbb{R}$ be a stable unfolding of immersions. If the equation is non-degenerated at $\left(t_{0}, x_{0}, y_{0}\right)$, then there exists an unfolding of immersions $\mathcal{I}^{\prime}$ such that $\widehat{\text { Image } \mathcal{I}^{\prime}}$ is a local geometric solution of $E\left(1, a_{1}, \ldots, a_{n} . b\right)$ and $\mathcal{I}, \mathcal{I}^{\prime}$ are $t$-P-A-equivalent.

Proof. Without loss of generality, we assume that $\left(t_{0}, x_{0}, y_{0}\right)=(0,0,0)$. Let $G$ : $\left(\mathbb{R} \times \mathbb{R}^{n} \times \mathbb{R}, 0\right) \rightarrow(\mathbb{R}, 0)$ be a generating family of the unfolding of immersion $\mathcal{I}$. Since $g=G \mid 0 \times \mathbb{R}^{n} \times \mathbb{R}$ is submersive, the set $g^{-1}(0)$ is the initial condition for the corresponding (GCPT). By the arguments of the characteristic method, we can construct a unfolding of immersions $I^{\prime}$ which is the local unique geometric solution around $g^{-1}(0)$.

We now choose a generating family $F:\left(\mathbb{R} \times \mathbb{R}^{n} \times \mathbb{R}, 0\right) \rightarrow(\mathbb{R}, 0)$ of $\mathcal{I}^{\prime}$. By definition, $F^{-1}(0) \mid\{0\} \times \mathbb{R}^{n} \times \mathbb{R}$ is equal to $g^{-1}(0)$, then we may assume that $f, g$ are $P$ - $\mathcal{K}$-equivalent, where $f=F \mid\{0\} \times \mathbb{R}^{n} \times \mathbb{R}$.

If $P-\mathcal{K}-\operatorname{cod} g=0$, then $P-\mathcal{K}-\operatorname{cod} f=0$, so that $f$ is already a $P$ - $\mathcal{K}$-versal deformation of itself. Hence, for the same reason as in the proof of Theorem $4.2, F$ is $t$ - $P$ - $\mathcal{K}$-equivalent to $G$.

We now assume that $P-\mathcal{K}-\operatorname{cod} g=1$, so that $P-\mathcal{K}-\operatorname{cod} f=1$. If $\left.\frac{\partial F}{\partial t}\right|_{t=0} \notin$ $T_{e}(P-\mathcal{K})(f)$, then we can get the required assertion by the uniqueness of the $P-\mathcal{K}$ versal deformation as in the previous case.

Suppose that $\left.\frac{\partial F}{\partial t}\right|_{t=0} \in T_{e}(P-\mathcal{K})(f)$ for any generating family $F$ of $\mathcal{I}^{\prime}$. Since $F^{-1}(0)$ is a geometric solution of $E\left(1, a_{1}, \ldots, a_{n}, b\right)$, we have a relation

$$
\frac{\partial F}{\partial t}+\sum_{i=1}^{n} a_{i}(t, x, y) \frac{\partial F}{\partial x_{i}}+b(t, x, y) \frac{\partial F}{\partial y}=0 \text { on } F^{-1}(0)
$$

so that

$$
-\frac{\partial F}{\partial t} \equiv \sum_{i=1}^{n} a_{i}(t, x, y) \frac{\partial F}{\partial x_{i}}+b(t, x, y) \frac{\partial F}{\partial y} \bmod \langle F\rangle_{\mathcal{\varepsilon}_{(t, x, y)}} .
$$

It follows that that

$$
-\left.\frac{\partial F}{\partial t}\right|_{t=0} \equiv \sum_{i=1}^{n} a_{i}(0, x, y) \frac{\partial f}{\partial x_{i}}+b(0, x, y) \frac{\partial f}{\partial y} \bmod \langle f\rangle_{\mathcal{E}_{(x, y)}}
$$

Therefore

$$
-\left.\frac{\partial F}{\partial t}\right|_{t=x=0} \equiv \sum_{i=1}^{n} a_{i}(0,0, y) \frac{\partial f}{\partial x_{i}}(0, y)+b(0,0, y) \frac{\partial f_{0}}{\partial y} \bmod \left\langle f_{0}\right\rangle_{\mathcal{E}_{y}}
$$

where $f_{0}(y)=f(0, y)$. We may assume that $f_{0} \in \mathfrak{M}_{y}^{k}$ for $k \geq 3$, where $\mathfrak{M}_{y}$ is the unique maximal ideal of $\mathcal{E}_{y}$. 
We now consider the Taylor polynomial of $a_{i}(t, x, y)$ for sufficiently higher order at $(t, x, 0)$ with respect to $y$-variables as follows:

$$
a_{i}(t, x, y)=a_{i}(t, x, 0)+\frac{\partial a_{i}}{\partial y}(t, x, 0) y+\frac{1}{2} \frac{\partial^{2} a_{i}}{\partial y^{2}}(t, x, 0) y^{2}+\text { higher terms. }
$$

On the other hand

$$
-\left.\frac{\partial F}{\partial t}\right|_{t=0} \in T_{e}(P-\mathcal{K})(f)=\left\langle f(x, y), \frac{\partial f}{\partial y}(x, y)\right\rangle_{\mathcal{E}_{(x, y)}}+\left\langle\frac{\partial f}{\partial x_{1}}(x, y), \ldots, \frac{\partial f}{\partial x_{n}}(x, y)\right\rangle_{\mathcal{E}_{x}} .
$$

It follows that

$$
\begin{aligned}
\sum_{i=1}^{n} a_{i}(0,0, y) & \frac{\partial f}{\partial x_{i}}(0, y)+b(0,0, y) \frac{\partial f_{0}}{\partial y}(y) \\
& \in\left\langle f_{0}(y), \frac{\partial f_{0}}{\partial y}(y)\right\rangle_{\mathcal{E}_{y}}+\left\langle\frac{\partial f}{\partial x_{1}}(0, y), \ldots, \frac{\partial f}{\partial x_{n}}(0, y)\right\rangle_{\mathbb{R}}
\end{aligned}
$$

and

$$
\begin{aligned}
\sum_{i=1}^{n}\left(a_{i}(0,0,0)\right. & \left.+\frac{\partial a_{i}}{\partial y}(0,0,0) y+\frac{1}{2} \frac{\partial^{2} a_{i}}{\partial y^{2}}(0,0,0) y^{2}+\text { higher terms }\right) \frac{\partial f}{\partial x_{i}}(0, y) \\
& \in\left\langle f_{0}(y), \frac{\partial f_{0}}{\partial y}(y)\right\rangle_{\varepsilon_{y}}+\left\langle\frac{\partial f}{\partial x_{1}}(0, y), \ldots, \frac{\partial f}{\partial x_{n}}(0, y)\right\rangle_{\mathbb{R}}
\end{aligned}
$$

For any linear isomorphism $A: \mathbb{R}^{n} \rightarrow \mathbb{R}^{n}$, we have a relation

$$
\frac{\partial f(A x, y)}{\partial x_{i}}=\sum_{j=1}^{n} A_{i j} \frac{\partial f}{\partial x_{j}}(A x, y)
$$

We remark that the vector space

$$
\left\langle f_{0}, \frac{\partial f_{0}}{\partial y}\right\rangle_{\mathcal{E}_{y}}+\left\langle\frac{\partial f}{\partial x_{1}}(0, y), \ldots, \frac{\partial f}{\partial x_{n}}(0, y)\right\rangle_{\mathbb{R}}
$$

is an invariant under the action of the linear isomorphism $A$. Since

$$
\sum_{i=1}^{n} a_{i}(0,0,0) \frac{\partial f}{\partial x_{i}}(0, y) \in\left\langle\frac{\partial f}{\partial x_{1}}(0, y), \ldots, \frac{\partial f}{\partial x_{n}}(0, y)\right\rangle_{\mathbb{R}}
$$

we have

$$
\begin{aligned}
& \left(\sum_{i=1}^{n} \frac{\partial a_{i}}{\partial y}(0,0,0) \frac{\partial f}{\partial x_{i}}(0, y)\right) y+\text { higher terms } \\
& \qquad\left\langle f_{0}, \frac{\partial f_{0}}{\partial y}\right\rangle_{\mathcal{E}_{y}}+\left\langle\frac{\partial f}{\partial x_{1}}(0, y), \ldots, \frac{\partial f}{\partial x_{n}}(0, y)\right\rangle_{\mathbb{R}}
\end{aligned}
$$


By assumptions, $\frac{\partial a_{i}}{\partial y}(0,0,0) \neq 0$ and $\frac{\partial f}{\partial x_{j}}(0,0) \neq 0$ for some $i, j=1, \ldots, n$. If necessary, by applying some linear isomorphism $A$, we have

$$
\sum_{i=1}^{n} \frac{\partial a_{i}}{\partial y}(0,0,0) \frac{\partial f}{\partial x_{i}}(0,0) \neq 0
$$

It follows that $\mathfrak{M}_{y} \subset\left\langle f_{0}, \frac{\partial f_{0}}{\partial y}\right\rangle_{\mathcal{E}_{y}}+\left\langle\frac{\partial f}{\partial x_{1}}(0, y), \ldots, \frac{\partial f}{\partial x_{n}}(0, y)\right\rangle_{\mathbb{R}} \bmod \mathfrak{M}_{y}^{2}$.

Since $\mathcal{K}$-cod $\left(f_{0}\right)$ is finite (for the definition of $\mathcal{K}$-finiteness, see $[10,13]$ ), then there exists $k \in \mathbb{N}$ such that $\mathfrak{M}_{y}^{k} \subset\left\langle f_{0}, \frac{\partial f_{0}}{\partial y}\right\rangle_{\mathcal{E}_{y}} \subset \mathfrak{M}_{y}^{k-1}$. If $y \in\left\langle f_{0}, \frac{\partial f_{0}}{\partial y}\right\rangle_{\mathcal{E}_{y}}$, we have $\mathfrak{M}_{y}=\left\langle f_{0}, \frac{\partial f_{0}}{\partial y}\right\rangle_{\mathcal{E}_{y}}$. Then this case is corresponding to the case $k=1$. We may assume that $y \in\left\langle\frac{\partial f}{\partial x_{1}}(0, y), \ldots, \frac{\partial f}{\partial x_{n}}(0, y)\right\rangle_{\mathbb{R}}$. It follows that there exist real numbers $\lambda_{i}(i=1, \ldots, n)$ such that $y=\sum_{i=1}^{n} \lambda_{i} \frac{\partial f}{\partial x_{i}}(0, y)$. If necessary, applying a linear isomorphism $A$, we may assume that $y=\frac{\partial f}{\partial x_{i}}(0, y)$ for some $i=1, \ldots, n$. By the same arguments as those of previous paragraphs, we can asserts that $y^{2} \epsilon$ $\left\langle f_{0}, \frac{\partial f_{0}}{\partial y}\right\rangle_{\mathcal{E}_{y}}+\left\langle\frac{\partial f}{\partial x_{1}}(0, y), \ldots, \frac{\partial f}{\partial x_{n}}(0, y)\right\rangle_{\mathbb{B}}$. We can continue this procedure up to degree $k-1$. Eventually, every polynomial of degree $k-1$ is contained in the vector space

$$
\mathfrak{M}_{y} \subset\left\langle f_{0}, \frac{\partial f_{0}}{\partial y}\right\rangle_{\mathcal{E}_{y}}+\left\langle\frac{\partial f}{\partial x_{1}}(0, y), \ldots, \frac{\partial f}{\partial x_{n}}(0, y)\right\rangle_{\mathbb{R}} \bmod \mathfrak{M}_{y}^{r}
$$

Since $\mathfrak{M}_{y}^{k} \subset\left\langle f_{0}, \frac{\partial f_{0}}{\partial y}\right\rangle_{\mathcal{E}_{y}}$, we have

$$
\mathcal{E}_{y}=\left\langle f_{0}, \frac{\partial f_{0}}{\partial y}\right\rangle_{\mathcal{E}_{y}}+\left\langle\frac{\partial f}{\partial x_{1}}(0, y), \ldots, \frac{\partial f}{\partial x_{n}}(0, y)\right\rangle_{\mathbb{R}} .
$$

It follows that

$$
\mathcal{E}_{(x, y)}=T_{e}(P-\mathcal{K})(f)+\mathfrak{M}_{y} \mathcal{E}_{(x, y)}
$$

thus we have $E_{(x, y)}=T_{e}(P-\mathcal{K})(f)$ by the Malgrange preparation theorem. This contradicts to the fact that $P-\mathcal{K}-\operatorname{cod}(f)=1$.

By the classification in Theorem 3.3, the generic nontrivial bifurcations of singularities are given by the germs ${ }^{1} A_{k}(k=2, \ldots, n+1)$. Especially the first singularity appears in the form of ${ }^{1} A_{2}$, if the initial condition is smooth. We show that the ${ }^{1} A_{k}$ singularity appear at an $(k-1)$-non-degenerated point of the equation as follows :

Theorem 4.5. If an ${ }^{1} A_{k}$ singularity appears at a point $\left(t_{0}, x_{0}, y_{0}\right)$, then the equation $E\left(1, a_{1}, \ldots, a_{n}, b\right)$ is $s$-non-degenerated at $\left(t_{0}, x_{0}, y_{0}\right)$ for some $s=1, \ldots, k-1$.

Proof. The method of the proof is analogous to those of Example 4.3 and Theorem 4.4.

Without loss of generality, we assume that $\left(t_{0}, x_{0}, y_{0}\right)=(0,0,0)$. We consider the function germ $f\left(x_{1}, \ldots, x_{n}, y\right)=y^{k+1}+y^{k-1}\left( \pm x_{k}^{2} \pm \cdots \pm x_{n}^{2}\right)+\sum_{i=1}^{k-1} x_{i} y^{i-1}$, then we adopt the hypersurface $\phi_{f} \subset\left(\mathbb{R}^{n} \times \mathbb{R}, 0\right)$. as an initial condition for the (GCPT). We can get an unfolding of immersions $\mathcal{I}$ by the method of characteristics. Let $G\left(t, x_{1}, \ldots x_{n}, y\right)$ be a generating family of $\mathcal{I}$, then $g\left(x_{1}, \ldots, x_{n}\right)=G\left(0, x_{1}, \ldots x_{n}, y\right)$ 
satisfies that $\phi_{f}=g^{-1}(0)$. It follows that there exists $\lambda \in \mathcal{E}_{(x, y)}$ such that $\lambda(0) \neq 0$ and $f\left(x_{1} \ldots, x_{n}, y\right)=\lambda\left(x_{1}, \ldots, x_{n}, y\right) g\left(x_{1}, \ldots, x_{n}, y\right)$. Define a function germ

$$
F:\left(\mathbb{R} \times \mathbb{R}^{n} \times \mathbb{R}, 0\right) \rightarrow(\mathbb{R}, 0)
$$

by $F\left(t, x_{1} \ldots x_{n}, y\right)=\lambda\left(x_{1}, \ldots, x_{n}, y\right) G\left(t, x_{1}, \ldots, x_{n}, y\right)$, then we have $F^{-1}(0)=$ $G^{-1}(0)=$ Image $\mathcal{I}$ and $F\left(0, x_{1}, \ldots, x_{n}, y\right)=f\left(x_{1}, \ldots, x_{n}, y\right)$. It follows that

$$
\frac{\partial F}{\partial t}(t, x, y)+\sum_{i=1}^{n} a_{i}(t, x, y) \frac{\partial F}{\partial x_{i}}(t, x, y)+b(t, x, y) \frac{\partial F}{\partial y}(t, x, y)=0 \quad \text { on } F^{-1}(0),
$$

so that

$$
-\frac{\partial F}{\partial t} \equiv \sum_{i=1}^{n} a_{i}(t, x, y) \frac{\partial F}{\partial x_{i}}+b(t, x, y) \frac{\partial F}{\partial y}(t, x, y) \bmod \langle F\rangle_{\mathcal{E}_{(t, x, y)}} .
$$

It follows that

$$
-\left.\frac{\partial F}{\partial t}\right|_{t=0} \equiv \sum_{i=1}^{n} a_{i}(0, x, y) \frac{\partial f}{\partial x_{i}}+b(0, x, y) \frac{\partial f}{\partial y} \bmod \langle f\rangle_{\mathcal{E}_{(x, y)}} .
$$

We now consider the Taylor polynomial of $a_{i}(t, x, y)$ of sufficiently higher order at $(t, x, 0)$ with respect to $y$-variables as follows :

$$
a_{i}(t, x, y)=a_{i}(t, x, 0)+\frac{\partial a_{i}}{\partial y}(t, x, 0) y+\frac{1}{2} \frac{\partial^{2} a_{i}}{\partial y^{2}}(t, x, 0) y^{2}+\text { higher terms. }
$$

On the other hand, we have

$$
\frac{\partial f}{\partial x_{i}}= \begin{cases}1 & i=1 \\ y^{i-1} & 2 \leq i \leq k-1 \\ \pm 2 x_{i} y & k \leq i \leq n\end{cases}
$$

and

$$
\frac{\partial f}{\partial y}=(k+1) y^{k}+(k-1) y^{k-2}\left( \pm x_{k}^{2} \pm \cdots \pm x_{n}^{2}\right)+\sum_{i=2}^{k-1}(i-1) x_{i} y^{i-2}
$$

It follows that

$$
\begin{aligned}
-\left.\frac{\partial F}{\partial t}\right|_{t=0} & \equiv a_{1}(0, x, 0)+\frac{\partial a_{1}}{\partial y}(0, x, 0) y+\frac{1}{2} \frac{\partial^{2} a_{1}}{\partial y^{2}}(0, x, 0) y^{2}+\text { higher terms } \\
+ & \sum_{i=2}^{k-1}\left(a_{i}(0, x, 0)+\frac{\partial a_{i}}{\partial y}(0, x, 0) y+\frac{1}{2} \frac{\partial^{2} a_{i}}{\partial y^{2}}(0, x, 0) y^{2}\right) y^{i-1}+\text { higher terms } \\
+ & \sum_{i=k}^{n}\left(a_{i}(0, x, 0)+\frac{\partial a_{i}}{\partial y}(0, x, 0) y+\frac{1}{2} \frac{\partial^{2} a_{i}}{\partial y^{2}}(0, x, 0) y^{2}\right)\left( \pm 2 x_{i} y\right)+\text { higher terms } \\
& +b(0, x, y) \frac{\partial f}{\partial y}(x, y) \\
& \quad \bmod \left\langle f, \frac{\partial f}{\partial y}\right\rangle_{\mathcal{E}_{(x, y)}}+\mathfrak{M}_{(x, y)}^{k+2} .
\end{aligned}
$$


We can show that all elements of $\mathcal{E}_{(x, y)}$ except $c y^{k-1}$ are contained in $T_{e}(P-\mathcal{K})(f)$, where $c$ is a constant real number.

If the equation $E\left(1, a_{1}, \ldots, a_{n}, b\right)$ is not $(k-1)$-non-degenerated at the point $(0,0,0)$, then we have $\frac{\partial^{s} a_{i}}{\partial y^{s}}(0,0,0)=0$ for any $i=1, \ldots, n$ and $s=1, \ldots, k-1$. Then we have

$$
\sum_{i=1}^{k} \frac{1}{(k-i) !} \frac{\partial^{(k-i)} a_{i}}{\partial y^{(k-i)}}(0, x, 0) \in \mathfrak{M}_{x}
$$

This means that $\left.\frac{\partial F}{\partial t}\right|_{t=0} \in T_{e}(P-\mathcal{K})(f)$ by the above calculations. This formula show that $F$ cannot be a $P$ - $\mathcal{K}$-versal deformation of $f$. However the generating family of type ${ }^{1} A_{k}$ is a $P$ - $\mathcal{K}$-versal deformation of $f$. Then $F$ is not $t-P$ - $\mathcal{K}$-equivalent to germ of type ${ }^{1} A_{k}$. Since $F$ and $G$ are $t$ - $P-\mathcal{K}$-equivalent, $G$ is not $t$ - $P$ - $\mathcal{K}$-equivalent to germ of type ${ }^{1} A_{k}$.

On the other hand, let $H$ be a generating family of a geometric solution of $E\left(1, a_{1}, \ldots, a_{n}, b\right)$. Suppose that $H$ is $t$-P- $\mathcal{K}$-equivalent to the germ of ${ }^{1} A_{k}$, then there exist a diffeomorphism germ

$$
\Phi:\left(\mathbb{R} \times \mathbb{R}^{n} \times \mathbb{R}, 0\right) \rightarrow\left(\mathbb{R} \times \mathbb{R}^{n} \times \mathbb{R}, 0\right)
$$

of the form

$$
\Phi(t, x, y)=\left(\phi_{1}(t), \phi_{2}(t, x), \phi_{3}(t, x, y)\right)
$$

and an element $\lambda(t, x, y) \in \mathcal{E}_{(t, x, y)}$ such that $\lambda(0) \neq 0$ and $\lambda(t, x, y) H \circ \Phi(t, x, y)=$ $F(t, x, y)$, where

$$
F(t, x, y)=y^{k+1}+y^{k-1}\left(t \pm x_{k}^{2} \pm \cdots \pm x_{n}^{2}\right)+\sum_{i=1}^{k-1} x_{i} y^{i-1}(2 \leq k \leq n+1) .
$$

Thus we have $\lambda(0, x, y) H\left(0, \phi_{2}(0, x), \phi_{3}(0, x, y)\right)=f(x, y)$. Let $G$ be a generating family of the geometric solution which is constructed by the characteristic method from the initial data $\phi_{f}$. By the uniqueness of the geometric solution, we can assert that $H$ is $t$-P- $\mathcal{K}$-equivalent to $G$. However, by the previous arguments, $G$ cannot be $t$-P-K-equivalent to $F$. This contradicts to the assumption that $H$ is $t$-P-Kequivalent to $F$. Thus the unfolding of immersions of type ${ }^{1} A_{k}$ cannot be realized as a geometric solution of $E\left(1, a_{1}, \ldots, a_{n}, b\right)$ at the point $\left(t_{0}, x_{0}, y_{0}\right)$.

The following corollary of the above theorem shows that the first singularity appears at a non-degenerated point of the equation, if the initial condition is smooth.

Corollary 4.6. If an ${ }^{1} A_{2}$ singularity appears at a point $\left(t_{0}, x_{0}, y_{0}\right)$, then the equation $E\left(1, a_{1}, \ldots, a_{n}, b\right)$ is non-degenerated at $\left(t_{0}, x_{0}, y_{0}\right)$.

\section{SinglE CONSERVATION LAWS}

In this section we apply the previous results on first order quasi-linear partial differential equations to single conservation laws.

Here, we consider the equation of the form

$$
\frac{\partial y}{\partial t}+\sum_{i=1}^{n} \frac{\partial f_{i}(y)}{\partial x_{i}}=0
$$


where $f_{i}$ 's are smooth functions. This equation is rewritten as

$$
\frac{\partial y}{\partial t}+\sum_{i=1}^{n} \frac{d f_{i}}{d y}(y) \frac{\partial y}{\partial x_{i}}=0
$$

so that it is considered as $E\left(1, d f_{1} / d y(y), \ldots, d f_{n} / d y(y), 0\right)$. Set $a_{i}(y)=\frac{d f_{i}}{d y}(y)$, $1 \leq i \leq n$. Then the characteristic equation associated with $(\mathrm{P})$ through $\left(0, x_{0}\right)$ is given as follows:

$$
\left\{\begin{array}{l}
\frac{d x_{i}}{d t}(t)=a_{i}\left(y(t, x(t)), \quad x_{i}(0)=x_{i, 0}\right. \\
\frac{d y}{d t}(t, x(t)), \quad y(0, x(0))=\phi\left(x_{0}\right) .
\end{array}\right.
$$

The solution of the characteristic equation can be expressed by

$$
x(t)=x_{0}+t a\left(\phi\left(x_{0}\right)\right) \text { and } y(t, x(t))=y(0, x(0))=\phi\left(x_{0}\right) .
$$

Therefore we define the corresponding immersion

$$
\mathcal{I}[a, \phi]: \mathbb{R} \times \mathbb{R}^{n} \rightarrow \mathbb{R} \times \mathbb{R}^{n} \times \mathbb{R}
$$

by

$$
\mathcal{I}[a, \phi](t, u)=(t, u+t a(\phi(u)), \phi(u)),
$$

where $a=\left(a_{1}, \ldots, a_{n}\right)$. We also define mappings

$$
F[a, \phi]: \mathbb{R} \times \mathbb{R}^{n} \rightarrow \mathbb{R}^{n}
$$

by

$$
F[a, \phi](t, u)=u+t a(\phi(u))
$$

and

$$
f\left[a, \phi ; t_{0}\right]: \mathbb{R}^{n} \rightarrow \mathbb{R}^{n}
$$

by

$$
f\left[a, \phi ; t_{0}\right](u)=F[a, \phi]\left(t_{0}, u\right) .
$$

By the classical theory of characteristics, it follows that the classical solution of (C) is expressed by $y\left(t_{0}, x\right)=\phi\left(f\left[a, \phi ; t_{0}\right]^{-1}(x)\right)$ at $x$ which is not critical value of $f\left[a, \phi ; t_{0}\right]$. Then our problem is to study the singularities of $f\left[a, \phi ; t_{0}\right]$. We can calculate the Jacobian matrix $J\left(f\left[a, \phi ; t_{o}\right]\right)$ of $f\left[a, \phi ; t_{0}\right]$ at $u_{0}$ as follows:

$$
\left(\begin{array}{cccc}
1+c_{11} & c_{12} & \ldots & c_{1 n} \\
c_{21} & 1+c_{22} & \ldots & c_{2 n} \\
\vdots & \vdots & \ddots & \vdots \\
c_{n 1} & c_{n 2} & \ldots & 1+c_{n n}
\end{array}\right)
$$

where $c_{i j}=t_{0} \frac{d a_{i}}{d y}\left(\phi\left(u_{0}\right)\right) \frac{\partial \phi}{\partial u_{j}}\left(u_{0}\right)$.

By definition (C) is called $k$-non-degenerated at $\left(t_{0}, x_{0}, y_{0}\right)$ if $\frac{d^{k+1} f}{d y^{k+1}}(y) \neq 0$ at $\left(t_{0}, x_{0}, y_{0}\right)$, where $\frac{d^{s} f}{d y^{s}}(y)=\left(\frac{d^{s} f_{1}}{d y^{s}}(y), \ldots, \frac{d^{s} f_{n}}{d y^{s}}(y)\right)$. Especially $(\mathrm{C})$ is said to be $n o n$ degenerated if it is 1-non-degenerated. Then we can summarize the corresponding results for general first order quasi-linear partial differential equations of evolution type to single conservation laws. 
Theorem 5.1. Let $\mathcal{I}:\left(R, u_{0}\right) \rightarrow\left(\mathbb{R} \times \mathbb{R}^{n} \times \mathbb{R},\left(t_{0}, x_{0}, y_{0}\right)\right)$ be a stable unfolding of immersions. Then

(1) If $\mathcal{I}$ has a trivial bifurcation, then there exists an unfolding of immersions $\mathcal{I}^{\prime}$ such that Image $\mathcal{I}^{\prime}$ is a local geometric solution of $E\left(1, a_{1}, \ldots, a_{n} . b\right)$ and $\mathcal{I}, \mathcal{I}^{\prime}$ are $t$-P-A-equivalent.

(2) If the equation $(C)$ is non degenerated at $\left(t_{0}, x_{0}, y_{0}\right)$, then there exists an unfolding of immersions $I^{\prime}$ such that Image $I^{\prime}$ is a local geometric solution of $(C)$ and $\mathcal{I}, \mathcal{I}^{\prime}$ are $t$-P-A-equivalent.

(3) If an ${ }^{1} A_{k}$ singularity appears at a point $\left(t_{0}, x_{0}, y_{0}\right)$, then the equation $(C)$ is non-degenerated at $\left(t_{0}, x_{0}, y_{0}\right)$.

Proof. The statements of (1), (2) are the same as those of Theorems 4.2 and 4.4. By Theorem 4.5, if an ${ }^{1} A_{k}$ singularity appears at a point $\left(t_{0}, x_{0}, y_{0}\right)=$ $\left(t_{0}, u_{0}+t_{0} a\left(\phi\left(u_{0}\right)\right), \phi\left(u_{0}\right)\right)$, then the equation (C) is $s$-non-degenerated at $\left(t_{0}, x_{0}, y_{0}\right)$ for some $s=1, \ldots, k-1$. However, if (C) is $s$-non-degenerated at $\left(t_{0}, x_{0}, y_{0}\right)$ for some $s \geq 2$, then $\frac{d a}{d y}\left(\phi\left(u_{0}\right)\right)=0$. It follows that the Jacobian matrix of $f\left[a, \phi ; t_{0}\right]$ is the unit matrix at $u_{0}$. So the point $\left(t_{0}, x_{0}, y_{0}\right)$ is not a singular point of the geometric solution. This contradicts to the fact that the geometric solution has ${ }^{1} A_{k}$ singularities at $\left(t_{0}, x_{0}, y_{0}\right)$.

In order to deal with generic properties for the Cauchy problem of single conservation laws $(P)$, we need a kind of the transversality theorems which is given in the Appendix. For notions and elementary properties we use hereafter, see Appendix. It follows from Theorems 3.3 and A.3 that the list of germs of map germs in the remark after Thoerem 3.3 gives a classification of map germs $f:\left(\mathbb{R}^{n}, 0\right) \rightarrow\left(\mathbb{R}^{n}, 0\right)$ with $\mathcal{A}-\operatorname{cod}(f) \leq 1$ and $\operatorname{dim} \operatorname{Ker} d f \leq 1$ :

$$
\left(u_{n}^{k+1}+\sum_{i=1}^{k-1} u_{i} u_{n}^{i}, u_{1}, \ldots, u_{n}\right)(1 \leq k \leq n)
$$

$\left({ }^{1} A_{k}\right)\left(u_{n}^{k+1}+u_{n}^{k-1}\left( \pm u_{k-1}^{2} \pm \cdots \pm u_{n-1}^{2}\right)+\sum_{i=1}^{k} u_{i} u_{n}^{i}, u_{1}, \ldots, u_{n}\right)(2 \leq k \leq n+1)$.

Let $J^{\ell}\left(\mathbb{R}^{n}, \mathbb{R}^{n}\right)$ be the $\ell$-jet space of mappings $\mathbb{R}^{n} \rightarrow \mathbb{R}^{n}$, where $\ell \geq n+2$. We consider a stratified subset $S=\left\{S_{j}\right\}_{j \in I}$ of $J^{\ell}\left(\mathbb{R}^{n}, \mathbb{R}^{n}\right)$ each strata is given by the $\mathcal{A}$-orbit of the $\ell$-jet of the germ in the above list. By Theorem B.1, we have the following theorem.

Theorem 5.2. There exists a residual subset $\mathcal{O} \subset C^{\infty}\left(\mathbb{R}^{n}, \mathbb{R}\right)$ with the following properties: For any $\phi \in \mathcal{O}$ and $\left(t_{0}, u_{0}\right) \in \mathbb{R} \times \mathbb{R}^{n}$, the germ $I[a, \phi]$ at $\left(t_{0}, u_{0}\right)$ is $t$-P-A-equivalent to one of germs in the list of Remarks after Theorem 3.3.

\section{MULTi-UNFOLDINGS OF IMMERSiONS}

As we discussed in the introduction, in order to study how the shock waves for the the entropy solution of $(\mathrm{P})$ evolve, we study the bifurcation of singularities appearing in different branches of the corresponding geometric solution. We classify 
the bifurcations of singularities of different branches by classifying the bifurcations of singularities of multi-unfoldings of immersions which are expressed in terms of multi-germs.

Let $\mathcal{I}_{i}:\left(R, u_{0}\right) \rightarrow\left(\mathbb{R} \times \mathbb{R}^{n} \times \mathbb{R},\left(0,0, y_{i}\right)(i=1, \ldots, r)\right.$ be unfoldings of immersions, where $y_{1}, \ldots, y_{r}$ are distinct. We call $\left(\mathcal{I}_{1}, \ldots, \mathcal{I}_{r}\right)$ a multi-unfolding of immersions. Let $\left(\mathcal{I}_{1}, \ldots, \mathcal{I}_{r}\right)$ and $\left(\mathcal{I}_{1}^{\prime}, \ldots, \mathcal{I}_{r}^{\prime}\right)$ be multi-unfoldings of immersions. We say that these are $t-(P-\mathcal{A})_{(r)}$-equivalent if there exist diffeomorphism germs

$$
\Phi_{i}:\left(\mathbb{R} \times \mathbb{R}^{n} \times \mathbb{R},\left(0,0, y_{i}\right) \rightarrow\left(\mathbb{R} \times \mathbb{R}^{n} \times \mathbb{R}\right),\left(0,0, y_{i}^{\prime}\right) \quad(i=1, \ldots, r)\right.
$$

of the form

$$
\Phi_{i}(t, x, y)=\left(\phi_{1}(t), \phi_{2}(t, x, y), \phi_{3}^{i}(t, x, y)\right)
$$

and a diffeomorphism germ $\Psi:\left(R, u_{0}\right) \rightarrow\left(R, u_{0}^{\prime}\right)$ such that $\Phi_{i} \circ \mathcal{I}_{i}=\mathcal{I}_{i}^{\prime} \circ \Psi$ for any $i=1, \ldots, r$. This equivalence describes how bifurcations of singularities of geometric solutions interact.

By the arguments in Section 3, there exist generating families $F_{i}:\left(\mathbb{R} \times \mathbb{R}^{n} \times\right.$ $\mathbb{R}, 0) \rightarrow(\mathbb{R}, 0)$ of $\mathcal{I}_{i}, i=1, \ldots, r$. We call $F=\left(F_{1}, \ldots, F_{r}\right)$ a multi-generating family of the multi-unfolding of immersions $\left(\mathcal{I}_{1}, \ldots, \mathcal{I}_{r}\right)$. We also consider an equivalence relation among multi-generating families of multi-unfoldings of immersions. Multi-generating families $F=\left(F_{1}, \ldots, F_{r}\right)$ and $F^{\prime}=\left(F_{1}^{\prime}, \ldots, F_{r}^{\prime}\right)$ are $t-(P-\mathcal{K})_{(r)^{-}}$ equivalent if there exists a diffeomorphism germ

$$
\Phi_{i}:\left(\mathbb{R} \times \mathbb{R}^{n} \times \mathbb{R}, 0\right) \rightarrow\left(\mathbb{R} \times \mathbb{R}^{n} \times \mathbb{R}, 0\right) \quad(i=1, \ldots, r)
$$

of the form

$$
\Phi_{i}(t, x, y)=\left(\phi_{1}(t), \phi_{2}(t, x, y), \phi_{3}^{i}(t, x, y)\right)
$$

such that

$$
\left\langle F_{i}^{\prime} \circ \Phi\right\rangle_{\mathcal{E}_{(t, x, y)}}=\left\langle F_{i}\right\rangle_{\mathcal{E}_{(\mathrm{t}, x, y)}} .
$$

We have the following simple proposition.

Proposition 6.1. Let $F=\left(F_{1}, \ldots, F_{r}\right)$ and $F^{\prime}=\left(F_{1}^{\prime}, \ldots, F_{r}^{\prime}\right)$ be multi-generating families of multi-unfoldings $\left(\mathcal{I}_{1}, \ldots, \mathcal{I}_{r}\right)$ and $\left(\mathcal{I}_{1}^{\prime}, \ldots, \mathcal{I}_{r}^{\prime}\right)$ respectively. Then $\left(\mathcal{I}_{1}, \ldots, \mathcal{I}_{r}\right)$ and $\left(\mathcal{I}_{1}^{\prime}, \ldots, \mathcal{I}_{r}^{\prime}\right)$ are $t-(P-\mathcal{A})_{(r)}$-equivalent if and only if $F$ and $F^{\prime}$ are $t-(P-\mathcal{K})_{(r)}$-equivalent.

According to the above proposition it is enough to give a classification of generic multi-generating families under the $(P-\mathcal{K})_{(r)}$-equivalence. For this we need to extend the results of the previous section to multi-generating families.

For generating families $F_{i}$ of $\Phi_{F_{i}}, i=1, \ldots, r$, we define a subspace of $\mathcal{E}_{(x, y)}^{r}$ by

$$
T_{e}(\mathrm{P}(r)-\mathcal{K})(f)=\left\langle\frac{\partial f_{1}}{\partial y}, f_{1}\right\rangle_{\mathcal{E}_{(x, y)}} \times \cdots \times\left\langle\frac{\partial f_{r}}{\partial y}, f_{r}\right\rangle_{\mathcal{E}_{(x, y)}}+\left\langle\frac{\partial f}{\partial x_{1}}, \ldots, \frac{\partial f}{\partial x_{n}}\right\rangle_{\mathcal{E}_{x}}
$$

and $(P-\mathcal{K})_{(r)}$ - $\operatorname{cod} f=\operatorname{dim}_{\mathbb{R}} \mathcal{E}_{(x, y)}^{r} / T_{e}\left((P-\mathcal{K})_{(r)}\right)(f)$, where $f_{i}=F_{i} \mid\{0\} \times \mathbb{R}^{n} \times \mathbb{R}$. We also say that $F$ is a $(P \cdot \mathcal{K})_{(r)}$-versal deformation of $f$ if

$$
\mathcal{E}_{(x, y)}^{r}=\left\langle\frac{\partial F}{\partial t} \mid\{0\} \times \mathbb{R}^{n} \times \mathbb{R}\right\rangle_{\mathbb{R}}+T_{e}\left((P-\mathcal{K})_{(r)}\right)(f) .
$$

By the versality theorem in [4], we have the following uniqueness result. 
Theorem 6.2. Let $F$ and $G$ be $(P-\mathcal{K})_{(r)}$-versal deformations of $f$ and $g$ respectively. Then $F$ and $G$ are $t-(P-\mathcal{K})_{(r)}$-equivalent if and only if $f$ and $g$ are $(P-\mathcal{K})_{(r)^{-}}$ equivalent.

Our objective is to classify multi-generating families $F=\left(F_{1}, \ldots, F_{r}\right)$ which are

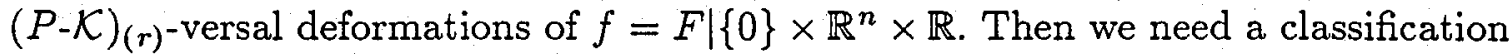
of multi-germs $f$ of $(P-\mathcal{K})_{(r)}$-cod $f \leq 1$. The following estimate of codimensions is useful for such a classification.

Lemma 6.3. $\sum_{i=1}^{r} \mathcal{K}-\operatorname{cod}\left(f_{0, i}\right) \leq(P-\mathcal{K})_{(r)}-\operatorname{cod}(f)+n$.

Here, $\mathcal{K}-\operatorname{cod}\left(f_{0, i}\right)=\operatorname{dim}_{\mathbb{R}} \mathcal{E}_{y} /\left\langle\frac{\partial f_{0, i}}{\partial y}, f_{0, i}\right\rangle_{\mathcal{E}_{y}}$.

Proof. We have $\mathcal{K}$-cod $\left(f_{0, i}\right)=\operatorname{dim}_{\mathbb{B}} \mathcal{E}_{(x, y)} /\left\langle\frac{\partial f_{i}}{\partial y}, f_{i}\right\rangle_{\mathcal{E}_{(x, y)}}+\mathfrak{M}_{x} \mathcal{E}_{(x, y)}$, so that

$$
\begin{aligned}
& \sum_{i=1}^{r} \mathcal{K}-\operatorname{cod}\left(f_{0, i}\right) \\
& \quad \operatorname{dim}_{\mathbb{R}} \mathcal{E}_{(x, y)}^{r} /\left\langle\frac{\partial f_{1}}{\partial y}, f_{1}\right\rangle_{\mathcal{E}_{(x, y)}} \times \cdots \times\left\langle\frac{\partial f_{r}}{\partial y}, f_{r}\right\rangle_{\mathcal{E}_{(x, y)}}+\mathfrak{M}_{x} \mathcal{E}_{(x, y)}^{r} \\
& \quad \leq \operatorname{dim}_{\mathbb{R}} \mathcal{E}_{(x, y)}^{r} / T_{e}(\mathrm{P}-\mathcal{K})_{(r)}(f) \\
& \quad+\operatorname{dim}_{\mathbb{R}} \frac{T_{e}\left((P-\mathcal{K})_{(r)}\right)(f)}{\left\langle\frac{\partial f_{1}}{\partial y}, f_{1}\right\rangle_{\mathcal{E}_{(x, y)}} \times \cdots \times\left\langle\frac{\partial f_{r}}{\partial y}, f_{r}\right\rangle_{\mathcal{E}_{(x, y)}}+\mathfrak{M}_{x} \mathcal{E}_{(x, y)}^{r}} \\
& \quad \leq \operatorname{dim}_{\mathbb{R}} \mathcal{E}_{(x, y)}^{r} / T_{e}\left((P-\mathcal{K})_{(r)}\right)(f)+\operatorname{dim}_{\mathbb{R}}\left\langle\frac{\partial \tilde{f}}{\partial x_{1}}, \ldots, \frac{\partial f}{\partial x_{n}}\right\rangle_{\mathbb{R}} \\
& \leq(P-\mathcal{K})_{(r)-\operatorname{cod}(f)+n .}
\end{aligned}
$$

We say that multi-function germs $f_{0}=\left(f_{0,1}, \ldots, f_{0, r}\right)$ and $g_{0}=\left(g_{0,1}, \ldots, g_{0, r}\right)$ are $\mathcal{K}_{(r)}$-equivalent if $f_{0, i}$ and $g_{0, i}$ are $\mathcal{K}$-equivalent for $i=1, \ldots, r$. For the definition and properties of the $\mathcal{K}$-equivalence, see [11]. We now define

$$
T_{e}(\mathcal{K})_{(r)}\left(f_{0}\right)=\left\langle\frac{\partial f_{0,1}}{\partial y}, f_{0,1}\right\rangle_{\mathcal{E}_{y}} \times \cdots \times\left\langle\frac{\partial f_{0, r}}{\partial y}, f_{0, r}\right\rangle_{\mathcal{E}_{y}} .
$$

We also define the notion of $\mathcal{K}_{(r)}$-versal deformation of $f_{0}$ as follows : Let $\bar{F}=$ $\left(\bar{F}_{1}, \ldots, \bar{F}_{r}\right)$ be a $s$-parameter deformation of $f_{0}$ (i.e. $\bar{F}_{i}:\left(\mathbb{R}^{s} \times \mathbb{R}, 0\right) \rightarrow(\mathbb{R}, 0)$ is a function germ such that $\bar{F}_{i} \mid\{0\} \times \mathbb{R}=f_{0, i}$ for any $i=1, \ldots, r$.) We say that $\bar{F}=\left(\bar{F}_{1}, \ldots, \bar{F}_{r}\right)$ is a $\mathcal{K}_{(r)}$-versal deformation of a multi-germ $f_{0}$ if

$$
\mathcal{E}_{y}=\left\langle\frac{\partial \bar{F}}{\partial u_{1}}\left|\{0\} \times \mathbb{R}, \ldots, \frac{\partial \bar{F}}{\partial u_{s}}\right|\{0\} \times \mathbb{R}\right\rangle_{\mathbb{R}}+T_{e}(\mathcal{K})_{(r)}\left(f_{0}\right) .
$$

We also define the discriminant set of $\bar{F}$ as follows:

$$
D_{\bar{F}}=\cup_{i=1}^{r} D_{\bar{F}_{i}},
$$

where

$$
D_{\bar{F}_{i}}=\left\{u \in \mathbb{R}^{s} \mid \bar{F}_{i}(u, y)=\frac{\partial \bar{F}_{i}}{\partial u_{1}}(u, y)=\cdots=\frac{\partial \bar{F}_{i}}{\partial u_{s}}(u, y)=0 \text { for some } y \in(\mathbb{R}, 0)\right\} \text {. }
$$

We have the following lemma. 
Lemma 6.4. If a multi-generating family $F$ is an one-parameter $(P-\mathcal{K})_{(r)}$-versal deformation of $f=F \mid\{0\} \times \mathbb{R}^{n} \times \mathbb{R}$, then it is an $(n+1)$-parameter $(\mathcal{K})_{(r)}$-versal deformation of $f_{0}=F \mid\{0\} \times\{0\} \times \mathbb{R}$.

We summarize the strategy for the classification, which will be presented in the next section, as follows :

Step 1. We classify multi-germs $f_{0}=\left(f_{0,1}, \ldots, f_{0, r}\right)$ with $(\mathcal{K})_{(r)}-\operatorname{cod}\left(f_{0}\right) \leq n+1$ under the $(\mathcal{K})_{(r)}$-equivalence, where $(\mathcal{K})_{(r)}-\operatorname{cod}\left(f_{0}\right)=\sum_{i=1}^{r} \mathcal{K}$-cod $\left(f_{0, i}\right)$.

Step 2. We construct $(n+1)$-parameter $(\mathcal{K})_{(r)}$-versal deformations $\bar{F}$ of the normal forms $f_{0}=\left(f_{0,1}, \ldots, f_{0, r}\right)$ obtained by Step 1 . We fix each germ $\bar{F}$ and we consider a smooth function germ $t:\left(\mathbb{R}^{n+1}, 0\right) \rightarrow(\mathbb{R}, 0)$. By the theorem in Zakalyukin [15, part 2.2], we can classify $t$ under coordinate changes of $\mathbb{R}^{n+2}$ which preserve the discriminant set $D_{\bar{F}}$. This classification generically corresponds to the classification of $F$ under the $(P-\mathcal{K})_{(r)}$-equivalence which preserves the projection on the $t$-space (i.e. the $t-(P-\mathcal{K})_{(r)}$-equivalence).

Step 3. We can check that each normal form obtained by Step 2 is a $(P-\mathcal{K})_{(r)^{-}}$ versal deformation when we consider $t$ as a parameter. Thus we can detect normal forms of generic multi-Legendrian unfoldings by the $P_{(r)}$-Legendrian equivalence.

\section{Classifications}

In this section we pursue the strategy we referred to at the end of the last section. Since we consider how singularities interact, we may assume that $\mathcal{K}$-cod $\left(f_{0, i}\right) \geq 1$ for a multi-germ $f_{0}=\left(f_{0,1}, \ldots, f_{0, r}\right)$. It follows from Lemma 6.3 that $r \leq n+1$. By Theorem 3.3, we have the following classification.

Lemma 7.1. For generic multi-generating families $F=\left(F_{1}, \ldots, F_{r}\right)$ of multiunfoldings of immersions, the corresponding multi-germs $f_{0}=\left(f_{0,1} \ldots, f_{0, r}\right)$ are $(\mathcal{K})_{(r)}$-equivalent to one of the multi-germs in the following list :

$$
\left(y^{k_{1}+1}, \ldots, y^{k_{r}+1}\right) \quad r \leq \sum_{i=1}^{r} k_{i} \leq n+1
$$

We can easily construct a $\mathcal{K}_{(r)}$-versal deformation for each multi-germ by the usual method. Then the corresponding list is as follows :

$$
\left(y^{k_{1}+1}+\sum_{i=1}^{k_{1}} u_{1, i} y^{i-1}, \ldots, y^{k_{r}+1}+\sum_{i=1}^{k_{r}} u_{r, i} y^{i-1}\right) \quad r \leq \sum_{i=1}^{r} k_{i} \leq n+1 .
$$

Let

$$
G\left(u_{1,1}, \ldots, u_{1, \mu_{1}}, \ldots, u_{r, 1}, \ldots, u_{r, \mu_{r}}, y\right)
$$

be a $\mathcal{K}_{(r)}$-versal deformation of a multi-germ $g=\left(g_{1}, \ldots, g_{r}\right)$, where $\mu_{i}=\mathcal{K}$-cod $\left.g_{i}\right)$ for $i=1, \ldots, r$. Define a multi-germ $\vec{G}$ by

$$
\begin{aligned}
\bar{G}\left(u_{1,1}, \ldots, u_{1, \mu_{1}}, \ldots, u_{r, 1}, \ldots, u_{r, \mu_{r}}, u_{1}, \ldots, u_{\mu}, y\right) \\
\quad=G\left(u_{1,1}, \ldots, u_{1, \mu_{1}}, \ldots, u_{r, 1}, \ldots, u_{r, \mu_{r}}, y\right)
\end{aligned}
$$

for $\mu=n+1-\sum_{i=1}^{r} \mu_{i}$. We now consider a function germ $t:\left(\mathbb{R}^{n+1}, 0\right) \rightarrow(\mathbb{R}, 0)$ on the $\left(u_{1,1}, \ldots, u_{1, \mu_{1}}, \ldots, u_{r, 1}, \ldots, u_{r, \mu_{r}}, u_{1}, \ldots, u_{\mu}\right)$-space. We can apply the theorem of part 2.2 in [15], so we get the following: 
Proposition 7.2. (Zakalyukin [15]) Suppose that

$$
\frac{\partial t}{\partial u_{1, \mu_{1}}} \neq 0, \ldots, \frac{\partial t}{\partial u_{r, \mu_{r}}} \neq 0
$$

and

$$
\left.t\right|_{u_{1,1}=\cdots=u_{r, \mu_{r}=0}} \text { is a Morse function germ. }
$$

Then there exists a diffeomorphism germ $\phi:\left(\mathbb{R}^{n+1}, 0\right) \rightarrow\left(\mathbb{R}^{n+1}, 0\right)$ preserving the discriminant set $D_{\bar{G}}$ such that $t \circ \phi$ is equal to $u_{1}$ or $\pm u_{1, \mu_{1}} \pm \cdots \pm u_{r, \mu_{r}} \pm\left(u_{1}\right)^{2} \pm$ $\cdots \pm\left(u_{\mu}\right)^{2}$.

We notice that a submersion germ $t:\left(\mathbb{R}^{n+1}, 0\right) \rightarrow(\mathbb{R}, 0)$ which satisfies the assumption of the proposition is generic. We can detect generic normal forms of multi-unfoldings of immersions as follows.

Theorem 7.3. The multi-generating family of a generic multi-unfolding of immersions is $t-(P-\mathcal{K})_{(r)}$-equivalent to one of the multi-germs in the following list:

$\left({ }^{0} A_{k_{1}, \ldots, k_{r}}\right)$

$$
\begin{array}{r}
\left(y^{k_{1}+1}+\sum_{i=1}^{k_{1}} x_{i} y^{i-1}, \ldots, y^{k_{r}+1}+\sum_{i=1}^{k_{r}} x_{\sum_{j=1}^{r-1} k_{j}+i} y^{i-1}\right) \\
\text { where } 1 \leq r \leq \sum_{j=1}^{r} k_{j} \leq n .
\end{array}
$$

$\left({ }^{1} A_{k}\right)$

$$
y^{k+1}+y^{k-1}\left(t \pm x_{k}^{2} \pm \cdots \pm x_{n}^{2}\right)+\sum_{i=1}^{k-1} x_{i} y^{i-1}
$$

where $2 \leq k \leq n+1$.

$\left({ }^{1} A_{k_{1}}^{0} A_{k_{2}, \ldots, k_{r}}\right)$

$$
\begin{array}{r}
\left(y^{k_{1}+1}+y^{k_{1}-1}\left(t+x_{k_{1}+k_{2}}+\cdots+x_{\sum_{j=1}^{s} k_{j}}+\cdots+x_{\ell}\right.\right. \\
\left. \pm x_{\ell+1}^{2} \pm \cdots \pm x_{n}^{2}\right)+\sum_{i=1}^{k_{1}-1} x_{i} y^{i-1} \\
\left.y^{k_{2}+1}+\sum_{i=1}^{k_{2}} x_{k_{1}+1} y^{i-1}, \ldots, y^{k_{r}+1}+\sum_{i=1}^{k_{1}} x_{\sum_{j=1}^{r-1} k_{j}+i} y^{i-1}\right) \\
\text { where } \ell=\sum_{j=1}^{r} k_{j}, 2 \leq r<\ell \leq n+1 .
\end{array}
$$

Proof. Let $G\left(u_{1,1}, \ldots, u_{1, \mu_{1}}, \ldots, u_{r, 1}, \ldots, u_{r, \mu_{r}}, y\right)$ be a $\mathcal{K}_{(r)}$-versal deformation of a multi-germ $g=\left(g_{1}, \ldots, g_{r}\right)$, where $\mu_{i}=\mathcal{K}$-cod $g_{i}$ for $i=1, \ldots, r$. Define a multigerm $\bar{G}$ by

$$
\begin{aligned}
\bar{G}\left(u_{1,1}, \ldots, u_{1, \mu_{1}}, \ldots, u_{r, 1}, \ldots,\right. & \left., u_{r, \mu_{r}}, u_{1}, \ldots, u_{\mu}, y\right) \\
& =G\left(u_{1,1}, \ldots, u_{1, \mu_{1}}, \ldots, u_{r, 1}, \ldots, u_{r, \mu_{r}}, y\right)
\end{aligned}
$$


for $\mu=n+1-\sum_{i=1}^{r} \mu_{i}$.

Let $F(t, x, y)$ be a multi-generating family of a $t$-(P-A $)_{(r)}$-stable multi-unfolding of immersions. Then $F$ is a $(P-\mathcal{K})_{(r)}$-versal deformation of a multi-germ $f=$ $F \mid\{0\} \times \mathbb{R}^{n} \times \mathbb{R}$, so that $F$ is a $(\mathcal{K})_{(r)}$-versal deformation of a multi-germ $f_{0}=$ $f \mid\{0\} \times \mathbb{R}$. If $f$ is $(\mathcal{K})_{(r)}$-equivalent to $g$, then $F$ and $\bar{G}$ are $(P-\mathcal{K})_{(r)}$-equivalent (i.e., there exist diffeomorphism germs

$$
\Psi_{i}:\left(\mathbb{R}^{n+1} \times \mathbb{R}, 0\right) \rightarrow\left(\left(\mathbb{R} \times \mathbb{R}^{n}\right) \times \mathbb{R}, 0\right) \quad(i=1, \ldots, r)
$$

of the form

$$
\Psi(u, q)=\left(\psi_{1}(u), \psi_{2}(u), \psi_{3}(u, y)\right)
$$

such that $\Psi^{*}\langle F\rangle_{\mathcal{E}_{(u, y)}}=\langle\bar{G}\rangle_{\mathcal{E}_{(u, y)}}$ ). By the remark after Proposition 7.2, we may assume that $\psi_{1}$ satisfies the assumption of Proposition 7.2 , so that there exists a diffeomorphism germ $\phi:\left(\mathbb{R}^{n+1}, 0\right) \rightarrow\left(\mathbb{R}^{n+1}, 0\right)$ preserving the discriminant set $\cup_{i=1}^{r} D_{\bar{G}_{i}}$ such that $\psi_{1} \circ \phi$ is equal to $u_{1}$ or $\pm u_{1, \mu_{1}} \pm \cdots \pm u_{r, \mu_{r}} \pm\left(u_{1}\right)^{2} \pm \cdots \pm\left(u_{\mu}\right)^{2}$. Here, the discriminant set $\cup_{i=1}^{r} D_{\bar{G}_{i}}$ is the wave front set of a multi-germ of a Legendrian submanifold in $P T^{*} \mathbb{R}^{n+1}$. Then we can construct the unique contact lift $\hat{\phi}$ of $\phi$ preserving the multi-germ of the Legendrian submanifold. That is, $(\phi \times$ $\left.1_{\mathbb{R}}\right)^{*} \bar{G}(u, y)=\bar{G}(\phi(u), y)$ gives the same multi-germ of the Legendrian submanifold given by $\bar{G}$. We can choose the coordinates of $\left(\mathbb{R} \times \mathbb{R}^{n}, 0\right)$ as follows

$$
\left\{\begin{array}{l}
t=\psi_{1} \circ \phi(u) \\
x=\psi_{2} \circ \phi(u)
\end{array}\right.
$$

where $u=\left(u_{1,1}, \ldots, u_{1, \mu_{1}}, \ldots, u_{r, 1}, \ldots, u_{r, \mu_{r}}, u_{1}, \ldots, u_{\mu}\right)$. By the above equality we can represent the coordinates $u$ by the coordinates $(t, x)$. This procedure gives the normal forms as follows: Let $G$ be a germ of the form

$$
\left(y^{k_{1}+1}+\sum_{i=1}^{k_{1}} u_{1, i} y^{i-1}, \ldots, y^{k_{r}+1}+\sum_{i=1}^{k_{r}} u_{r, i} y^{i-1}\right) \quad r \leq \sum_{i=1}^{r} k_{i} \leq n+1
$$

It follows from Proposition 7.2 that $t=u_{1}$ or $t= \pm u_{1, \mu_{1}} \pm \cdots \pm u_{r, \mu_{r}} \pm\left(u_{1}\right)^{2} \pm$ $\cdots \pm\left(u_{\mu}\right)^{2}$, where $\mu_{j}=k_{j}$ and $\mu=n+1-\sum_{j=1}^{r} k_{j}$. If $1 \leq r \leq \sum_{j=1}^{r} k_{j} \leq n$ and $t=u_{1}$, then we can use the coordinates

$$
u_{1, i}=x_{i},\left(1 \leq i \leq k_{1}\right) u_{2, i}=x_{k_{1}+i},\left(1 \leq i \leq k_{2}\right), \ldots, u_{r, i}=x_{\ell+i},\left(1 \leq i \leq k_{r}\right)
$$

where $\ell=\sum_{j=1}^{r-1} k_{j}$, so that we get the normal forms $\left({ }^{0} A_{k_{1}, \ldots, k_{r}}\right)$. If $r=1$ and $2 \leq k \leq n+1$, we already got the normal forms in Theorem 3.3. If $2 \leq r<$ $\sum_{j=1}^{r} k_{j} \leq n+1$ and $t= \pm u_{1, \mu_{1}} \pm \cdots \pm u_{r, \mu_{r}} \pm\left(u_{1}\right)^{2} \pm \cdots \pm\left(u_{\mu}\right)^{2}$, then we can detect the normal forms $\left({ }^{1} A_{k_{1}}^{0} A_{k_{2}, \ldots, k_{r}}\right)$ by the appropriate coordinate changes.

Finally, we can easily check that each normal form is $(P-\mathcal{K})_{(r)}$-versal. 


\section{APPENdix A. Stability of UNFoldings of IMMERSIONS}

Here we consider the stability of unfoldings of immersions by using the standard method of singularity theory. We refer the articles $[10,13]$ about this topics.

Let $\mathcal{I}:\left(\mathbb{R} \times \mathbb{R}^{n}, 0\right) \rightarrow\left(\mathbb{R} \times \mathbb{R}^{n} \times \mathbb{R}, 0\right)$ be an unfolding of immersions of the form $\mathcal{I}(t, u)=(t, x(t, u), y(t, u))$. By definition, we can choose coordinates such that the components of the unfolding of immersions is the above form. We say that $\mathcal{I}$ is infinitesimally $t$-P-A-stable if

$\mathcal{E}_{(t, u)}^{n+1}=\pi\left(\left\langle\left(1, \frac{\partial x}{\partial t}, \frac{\partial y}{\partial t}\right)\right\rangle_{\mathcal{E}_{t}}\right)+\left\langle\left(\frac{\partial x}{\partial u_{i}}, \frac{\partial y}{\partial u_{i}}|i=1, \ldots, n\rangle_{\mathcal{E}_{(t, u)}}+(t, x)^{*} \mathcal{E}_{(t, x)}^{n} \times \mathcal{I}^{*} \mathcal{E}_{(t, x, y)}\right.\right.$,

where $\pi: \mathcal{E}_{(t, u)}^{n+2} \rightarrow \mathcal{E}_{(t, u)}^{n+1}$ is the canonical projection defined by $\pi\left(h_{1}, \ldots, h_{n+2}\right)=$ $\left(h_{2}, \ldots, h_{n+2}\right)$ and $(t, x)^{*}, \mathcal{I}^{*}$ are the pull back homomorphisms defined as in Theorem 4.1 .

We can define the notion of $t$-P-A-stability and show that it is equivalent to the notion of infinitesimal stability. However, we do not need this fact so that we omit the details. We define map germs

$$
F[\mathcal{I}]:\left(\mathbb{R} \times \mathbb{R}^{n}, 0\right) \rightarrow\left(\mathbb{R}^{n}, 0\right)
$$

by

$$
F[\mathcal{I}](t, u)=x(t, u)
$$

and

$$
f[\mathcal{I}]:\left(\mathbb{R}^{n}, 0\right) \rightarrow\left(\mathbb{R}^{n}, 0\right)
$$

by

$$
f[\mathcal{I}](u)=x(0, u) .
$$

We say that $F[\mathcal{I}]$ is an infinitesimally $\mathcal{A}$-versal deformations of $f[\mathcal{I}]$ if

$$
\mathcal{E}_{u}^{n}=\left\langle\left.\frac{\partial F[\mathcal{I}]}{\partial t}\right|_{t=0}\right\rangle_{\mathbb{R}}+\left\langle\frac{\partial f[\mathcal{I}]}{\partial u_{i}} \mid i=1, \ldots, n\right\rangle_{\mathcal{E}_{u}}+f[\mathcal{I}]^{*} \mathcal{E}_{x}^{n}
$$

Proposition A.1. Under the above notations, the following are equivalent.

(1) $\mathcal{I}$ is infinitesimally $t-P$-A-stable.

(2) $F[\mathcal{I}]$ is an infinitesimally $\mathcal{A}$-versal deformations of $f[\mathcal{I}]$.

Proof. Suppose that $F[\mathcal{I}]$ is an infinitesimally $\mathcal{A}$-versal deformations of $f[\mathcal{I}]$. Since $\mathcal{I}_{0}=\mathcal{I} \mid\{0\} \times \mathbb{R}^{n}$ is an immersion, we have $\mathcal{I}_{0}^{*} \mathcal{E}_{(x, y)}=\mathcal{E}_{u}$ so that

$$
\begin{array}{r}
\mathcal{E}_{u}^{n+1}=\left(\left\langle\left.\frac{\partial F[\mathcal{I}]}{\partial t}\right|_{t=0}\right\rangle_{\mathbb{R}}+\left\langle\frac{\partial f[\mathcal{I}]}{\partial u_{i}} \mid i=1, \ldots, n\right\rangle_{\mathcal{E}_{u}}+f[\mathcal{I}]^{*} \mathcal{E}_{x}^{n}\right) \times \mathcal{I}_{0}^{*} \mathcal{E}_{(x, y)}= \\
\left.\left\langle\left(\left.\frac{\partial F[\mathcal{I}]}{\partial t}\right|_{t=0},\left.\frac{\partial y}{\partial t}\right|_{t=0}\right)\right\rangle_{\mathbb{R}}+\left\langle\left(\frac{\partial f[\mathcal{I}]}{\partial u_{i}},\left.\frac{\partial y}{\partial u_{i}}\right|_{t=0}\right) \mid i=1, \ldots, n\right\rangle_{\mathcal{E}_{u}}+f[\mathcal{I}]^{*} \mathcal{E}_{x}^{n}\right) \times \mathcal{I}_{0}^{*} \mathcal{E}_{(x, y)} .
\end{array}
$$

It follows that

$$
\begin{aligned}
\mathcal{E}_{(t, u)}^{n+1}=\pi\left(\left\langle\left(1, \frac{\partial x}{\partial t}, \frac{\partial y}{\partial t}\right)\right\rangle_{\mathcal{E}_{t}}\right) & +\left\langle\left(\frac{\partial x}{\partial u_{i}}, \frac{\partial y}{\partial u_{i}}\right) \mid i=1, \ldots, n\right\rangle_{\mathcal{E}_{(t, u)}} \\
& +(t, x)^{*} \mathcal{E}_{(t, x)}^{n} \times \mathcal{I}^{*} \mathcal{E}_{(t, x, y)}+\mathfrak{M}_{t} \mathcal{E}_{(t, u)}^{n+1}
\end{aligned}
$$


By the generalized Malgrange preparation theorem[14], we have $\mathcal{E}_{(t, u)}^{n+1}=\pi\left(\left\langle\left(1, \frac{\partial x}{\partial t}, \frac{\partial y}{\partial t}\right)\right\rangle_{\mathcal{E}_{t}}\right)+\left\langle\left(\frac{\partial x}{\partial u_{i}}, \frac{\partial y}{\partial u_{i}}|i=1, \ldots, n\rangle_{\mathcal{E}_{(t, u)}}+(t, x)^{*} \mathcal{E}_{(t, x)}^{n} \times \mathcal{I}^{*} \mathcal{E}_{(t, x, y)}\right.\right.$

The converse direction of the proof is given by the direct arguments.

Our purpose in here is to give a characterization of the stability in terms of generating families. Let $F_{\mathcal{I}}:\left(\mathbb{R} \times \mathbb{R}^{n} \times \mathbb{R}, 0\right) \rightarrow(\mathbb{R}, 0)$ be a generating family of an unfolding of immersions $\mathcal{I}:\left(\mathbb{R} \times \mathbb{R}^{n}, 0\right) \rightarrow\left(\mathbb{R} \times \mathbb{R}^{n} \times \mathbb{R}, 0\right)$. Then we have the following characterization theorem.

Theorem A.2. Under the above notations, the following are equivalent.

(1) $\mathcal{I}$ is infinitesimally $t-P$-A-stable.

(2) $F_{\mathcal{I}}$ is the $P$-K -versal deformation of $f_{\mathcal{I}}$, where $f_{\mathcal{I}}=F_{\mathcal{I}} \mid\{0\} \times \mathbb{R}^{n} \times \mathbb{R}$.

In order to prove the theorem, we need some preparations. For a given function germ $G\left(t, u_{1}, \ldots, u_{r}, v\right)$, we define map germs

$$
\tilde{G}:\left(\mathbb{R} \times \mathbb{R}^{r} \times \mathbb{R}, 0\right) \rightarrow(\mathbb{R}, 0)
$$

by

$$
G\left(t, u_{1}, \ldots, u_{r-1}, v\right)-u_{r}
$$

where $\left(t, u_{1}, \ldots, u_{r}, v\right) \in\left(\mathbb{R} \times \mathbb{R}^{r} \times \mathbb{R}, 0\right)$ and

$$
\mathcal{I}_{G}:\left(\mathbb{R} \times \mathbb{R}^{r}, 0\right) \rightarrow\left(\mathbb{R}^{r}, 0\right)
$$

by

$$
\mathcal{I}_{G}\left(t, u_{1}, \ldots, u_{r}, v\right)=\left(u_{1}, \ldots, u_{r-1}, G\left(t, u_{1}, \ldots, u_{r-1}, v\right)\right) .
$$

We also define a vector space

$$
T_{e}(\mathcal{A})\left(\mathcal{I}_{g}\right)=\left\langle\frac{\partial \mathcal{I}_{g}}{\partial u_{i}}, \frac{\partial \mathcal{I}_{g}}{\partial v} \mid i=1, \ldots r-1\right\rangle_{\mathcal{E}_{\left(u^{\prime}, v\right)}}+\mathcal{I}_{g}^{*} \mathcal{E}_{u}^{r}
$$

where $\mathcal{I}_{g}=\mathcal{I}_{G} \mid\{0\} \times \mathbb{R}^{r}$ and $u^{\prime}=\left(u_{1}, \ldots, u_{r-1}\right)$. Then we have the following lemma.

Lemma. Under the above notations, the following are equivalent.

(1) $\tilde{G}$ is the P-X-versal deformation of $\tilde{g}$, where $\tilde{g}=\tilde{G} \mid\{0\} \times \mathbb{R}^{r} \times \mathbb{R}$.

(2) $\mathcal{I}_{G}$ is the infinitesimally $\mathcal{A}$-versal deformation of $\mathcal{I}_{g}$.

Proof. By definition, we have

$$
T_{e}(\mathrm{P}-\mathcal{K})(\tilde{g})=\left\langle\frac{\partial g}{\partial v}, \tilde{g}\right\rangle_{\mathcal{E}_{(u, v)}}+\left\langle\frac{\partial g}{\partial u_{1}}, \ldots, \frac{\partial g}{\partial u_{r-1}}, 1\right\rangle_{\mathcal{E}_{u}}
$$

We clefine a map germ

$$
\tilde{\mathcal{I}}_{g}:\left(\mathbb{R}^{r}, 0\right) \rightarrow\left(\mathbb{R}^{r} \times \mathbb{R}, 0\right)
$$

by

$$
\tilde{\mathcal{I}}_{g}\left(u_{1}, \ldots, u_{r-1}, v\right)=\left(u_{1}, \ldots, u_{r-1}, g\left(, u_{1}, \ldots, u_{r-1}, v\right), v\right)
$$


We consider the pull back homomorphism

$$
\tilde{\mathcal{I}}_{g}^{*}: \mathcal{E}_{(u, v)} \rightarrow \mathcal{E}_{\left(u^{\prime}, v\right)}
$$

then we have $\operatorname{Ker} \tilde{\mathcal{I}}_{g}^{*}=\left\langle g\left(u_{1}, \ldots, u_{r-1}\right)-u_{r}\right\rangle_{\mathcal{E}_{(u, v)}}$. It is easy to show that

$$
\tilde{\mathcal{I}}_{g}^{*}\left(T_{e}(\mathrm{P}-\mathcal{K})(\tilde{g})\right)=\left\langle\frac{\partial g}{\partial v}\right\rangle_{\mathcal{E}_{\left(u^{\prime}, v\right)}}+\left\langle\frac{\partial g}{\partial u_{1}}, \ldots, \frac{\partial g}{\partial u_{r-1}}, 1\right\rangle_{\tilde{\mathcal{I}}_{\dot{g}}^{*}\left(\mathcal{E}_{u}\right)} .
$$

Furthermore, we have $\tilde{\mathcal{I}}_{g}^{*}\left(\mathcal{E}_{u}\right)=\mathcal{I}_{g}^{*}\left(\mathcal{E}_{u}\right)$,

$$
\left(\tilde{\mathcal{I}}_{g}^{*}\right)^{-1}\left(\left\langle\frac{\partial g}{\partial v}\right\rangle_{\mathcal{E}_{\left(u^{\prime}, v\right)}}+\left\langle\frac{\partial g}{\partial u_{1}}, \ldots, \frac{\partial g}{\partial u_{r-1}}, 1\right\rangle_{\tilde{\mathcal{I}}_{g}^{*}\left(\mathcal{E}_{u}\right)}\right)=T_{e}(\mathrm{P}-\mathcal{K})(\tilde{g})
$$

and $\tilde{\mathcal{I}}_{g}^{*}\left(\left\langle\frac{\partial \tilde{G}}{\partial t} \mid\{0\} \times \mathbb{R}^{r} \times \mathbb{R}\right\rangle_{\mathbb{R}}\right)=\left\langle\frac{\partial G}{\partial t} \mid\{0\} \times \mathbb{R}^{r}\right\rangle_{\mathbb{R}}$. It follows that $\tilde{G}$ is the P-K versal deformation of $\tilde{g}$ if and only if the following condition holds:

$$
\mathcal{E}_{\left(u^{\prime}, v\right)}=\left\langle\frac{\partial G}{\partial t} \mid\{0\} \times \mathbb{R}^{r}\right\rangle_{\mathbb{R}}^{\prime}+\left\langle\frac{\partial g}{\partial v}\right\rangle_{\mathcal{E}_{\left(u^{\prime}, v\right)}}+\left\langle\frac{\partial g}{\partial u_{1}}, \ldots, \frac{\partial g}{\partial u_{r-1}}, 1\right\rangle_{\tilde{I}_{g}^{*}\left(\mathcal{E}_{u}\right)} .
$$

On the other hand, we consider the subring $\{0\} \times \mathcal{E}_{\left(u^{\prime}, v\right)} \subset \mathcal{E}_{\left(u^{\prime}, v\right)}^{r}$, where $0 \in$ $\mathcal{E}_{\left(u^{\prime}, v\right)}^{r-1}$. It is easy to show that

$$
\begin{aligned}
\{0\} \times \mathcal{E}_{\left(u^{\prime}, v\right)} & \cap\left(\left\langle\frac{\partial \mathcal{I}_{G}}{\partial t} \mid\{0\} \times \mathbb{R}^{r}\right\rangle_{\mathbb{R}}+T_{e}(\mathcal{A})\left(\mathcal{I}_{g}\right)\right) \\
& =\{0\} \times\left(\left\langle\frac{\partial G}{\partial t} \mid\{0\} \times \mathbb{R}^{r}\right\rangle_{\mathbb{R}}+\left\langle\frac{\partial g}{\partial v}\right\rangle_{\mathcal{E}_{\left(u^{\prime}, v\right)}}+\left\langle\frac{\partial g}{\partial u_{1}}, \ldots, \frac{\partial g}{\partial u_{r-1}}, 1\right\rangle_{\tilde{I}_{g}^{*}\left(\mathcal{E}_{u}\right)}\right) .
\end{aligned}
$$

Since $\{0\} \times \mathcal{E}_{\left(u^{\prime}, v\right)}+T_{e}(\mathcal{A})\left(\mathcal{I}_{g}\right)=\mathcal{E}_{\left(u^{\prime}, v\right)}^{r}$, we can show that the following conditions are equivalent:

$$
\begin{gathered}
\mathcal{E}_{\left(u^{\prime}, v\right)}=\left\langle\frac{\partial G}{\partial t} \mid\{0\} \times \mathbb{R}^{r}\right\rangle_{\mathbb{R}}+\left\langle\frac{\partial g}{\partial v}\right\rangle_{\mathcal{E}_{\left(u^{\prime}, v\right)}}+\left\langle\frac{\partial g}{\partial u_{1}}, \ldots, \frac{\partial g}{\partial u_{r-1}}, 1\right\rangle_{\tilde{I}_{g}^{*}\left(\mathcal{E}_{u}\right)} \\
\mathcal{E}_{\left(u^{\prime}, v\right)}^{r}=\left\langle\frac{\partial \mathcal{I}_{G}}{\partial t} \mid\{0\} \times \mathbb{R}^{r}\right\rangle_{\mathbb{R}}+T_{e}(\mathcal{A})\left(\mathcal{I}_{g}\right) .
\end{gathered}
$$

This competes the proof of Lemma.

Proof of Theorem A.2. By Proposition A.1, it is enough to show that the condition (2) is equivalent to the fact that $F[\mathcal{I}]$ is an infinitesimally $\mathcal{A}$-versal deformation of $f[\mathcal{I}]$. If $\frac{\partial F_{I}}{\partial y} \neq 0$ at 0 , then there exists a function germ $G\left(t, x_{1}, \ldots, x_{n}\right)$ such that

$$
F_{\mathcal{I}}^{-1}(0)=\left\{\left(t, x_{1}, \ldots, x_{n}, G\left(t, x_{1}, \ldots, x_{n}\right)\right) \mid\left(t, x_{1}, \ldots, x_{n}\right) \in\left(\mathbb{R} \times \mathbb{R}^{n}, 0\right)\right\} .
$$

In this case, we may consider that

$$
\mathcal{I}\left(t, x_{1}, \ldots, x_{n}\right)=\left(t, x_{1}, \ldots, x_{n}, G\left(t, x_{1}, \ldots, x_{n}\right)\right)
$$


so that $F[\mathcal{I}]\left(t ; x_{1}, \ldots, x_{n}\right)=\left(x_{1}, \ldots, x_{n}\right)$. Thus $f[\mathcal{I}]$ is the $\mathcal{A}$-versal deformation of itself. Of course $F[\mathcal{I}]$ is also the $\mathcal{A}$-versal deformation of $f[\mathcal{I}]$.

On the other hand, the corresponding generating family is given by

$$
F_{\mathcal{I}}\left(t, x_{1}, \ldots, x_{n}, y\right)=G\left(t, x_{1}, \ldots, x_{n}\right)-y
$$

so that we have

$$
T_{e}(\mathrm{P}-\mathcal{K})\left(f_{\mathcal{I}}\right)=\left\langle 1, g\left(x_{1}, \ldots, x_{n}\right)-y\right\rangle_{\mathcal{E}_{(x, y)}}+\left\langle\frac{\partial g}{\partial x_{1}}, \ldots, \frac{\partial g}{\partial x_{n}}\right\rangle_{\mathcal{E}_{x}}=\mathcal{E}_{(x, y)},
$$

where $g\left(x_{1}, \ldots, x_{n}\right)=G\left(0, x_{1}, \ldots, x_{n}\right)$.

In the case when $\frac{\partial F_{I}}{\partial y}=0$ at 0 , we may assume that $\frac{\partial F_{\tau}}{\partial x_{n}} \neq 0$. It follows from the implicit function theorem that the corresponding generating family may be given by

$$
F_{\mathcal{I}}\left(t, x_{1}, \ldots, x_{n}, y\right)=G\left(t, x_{1}, \ldots, x_{n-1}, y\right)-x_{n} .
$$

This case corresponds to the lemma if we put $r=n$ and $\left(t, u_{1}, \ldots, u_{n}, v\right)=$ $\left(t, x_{1}, \ldots, x_{n}, y\right)$. The assertion of the lemma gives the proof of Theorem A.2.

By the proof of the lemma, we also have the following proposition.

Proposition A.3. Under the same notations as those of Proposition A.1 and Theorem A.2, we have the following equality:

$$
\mathcal{A}-\operatorname{cod} f[\mathcal{I}]=P-\mathcal{K} \operatorname{cod} f_{\mathcal{I}}
$$

where $\mathcal{A}$-codf $[\mathcal{I}]=\operatorname{dim}_{\mathbb{R}} \mathcal{E}_{u} / T_{e}(\mathcal{A})(f[\mathcal{I}])$.

\section{APPENDIX B. A TRANSVERSALITy THEOREM}

Here we show a transversality theorem for mappings

$$
F[a, \phi]: \mathbb{R} \times \mathbb{R}^{n} \rightarrow \mathbb{R}^{n}
$$

defined by

$$
F[a, \phi](t, u)=u+t a(\phi(u)) .
$$

As an application of the transversality theorem, we have Theorem 5.2. Throughout the reminder of Appendix B, we fix the smooth mapping $a(y)=\left(a_{1}, \ldots, a_{n}(y)\right)$. Let $j^{\ell}\left(\mathbb{R}^{n}, \mathbb{R}^{n}\right)$ be the $\ell$-jet space of mappings $\mathbb{R}^{n} \rightarrow \mathbb{R}^{n}$, where $\ell \geq n+2$. We use notions and results in the theory of singularities of smooth mappings (cf.,[8],[11]). Let $f, g:\left(\mathbb{R}^{n}, 0\right) \rightarrow\left(\mathbb{R}^{n}, 0\right)$ be map germs. We say that $f$ and $g$ are $\mathcal{A}$-equivalent if there exist diffeomorphism germs $\phi, \psi:\left(\mathbb{R}^{n}, 0\right) \rightarrow\left(\mathbb{R}^{n}, 0\right)$ such that $\psi \circ f=g \circ \phi$. It follows that there exists an action of $\operatorname{Diff}\left(\mathbb{R}^{n}, 0\right) \times \operatorname{Diff}\left(\mathbb{R}^{n}, 0\right)$ on $J^{\ell}(n, n)$ induce by the $\mathcal{A}$-equivalence, where $\operatorname{Diff}\left(\mathbb{R}^{n}, 0\right)$ is the group of diffeomorphism germs of $\left(\mathbb{R}^{n}, 0\right)$ onto itself and we use the canonical decomposition $J^{\ell}\left(\mathbb{R}^{n}, \mathbb{R}^{n}\right)=\mathbb{R}^{n} \times \mathbb{R}^{n} \times J^{\ell}(n, n)$ :

$$
\Psi: \operatorname{Diff}\left(\mathbb{R}^{n}, 0\right) \times \operatorname{Diff}\left(\mathbb{R}^{n}, 0\right) \times J^{\ell}(n, n) \rightarrow J^{\ell}(n, n)
$$

by $\Psi\left((\psi, \phi), j_{0}^{\ell} f\right)=j_{0}^{\ell}\left(\psi \circ f \circ \phi^{-1}\right)$. We say that a subset $S \subset J^{\ell}(n, n)$ is $\mathcal{A}$-invariant if it is invariant under the above group action.

For any subset $S \subset J^{\ell}(n, n)$, we denote that $\hat{S}=\mathbb{R}^{n} \times \mathbb{R}^{n} \times S$ under the decomposition $J^{\ell}\left(\mathbb{R}^{n}, \mathbb{R}^{n}\right)=\mathbb{R}^{n} \times \mathbb{R}^{n} \times J^{\ell}(n, n)$. Let $C^{\infty}\left(\mathbb{R}^{n}, \mathbb{R}\right)$ be the space of $C^{\infty}$-functions equipped with Whitney $C^{\infty}$-topology. Then we have the following theorem. 
Theorem B.1. (A transversality theorem). Let $S$ be an $\mathcal{A}$-invariant submanifold of $J^{\ell}(n, n)$. Then

$$
T_{S}=\left\{\phi \in C^{\infty}\left(\mathbb{R}^{n}, \mathbb{R}\right) \mid j_{1}^{\ell} F[a, \phi] \text { is transversal to } \hat{S}\right\}
$$

is a residual subset of $C^{\infty}\left(\mathbb{R}^{n}, \mathbb{R}\right)$. Here, we define a map

$$
j_{1}^{\ell} F[a, \phi]: \mathbb{R} \times \mathbb{R}^{n} \rightarrow J^{\ell}\left(\mathbb{R}^{n}, \mathbb{R}^{n}\right)
$$

by

$$
j_{1}^{\ell} F[a, \phi](t, u)=j^{\ell} F_{t}[a, \phi](u),
$$

where $F_{t}[a, \phi](u)=F[a, \phi](t, u)$.

Moreover, if $S$ is closed subset, then $T_{S}$ is an open subset.

In order to prove the transversality theorem, we need some preparations. The second assertion of Theorem B.1 is follows directly from the following lemma.

Lemma B.2. Suppose that $S$ is a closed subset of $J^{\ell}(n, n)$. Then $T_{S}$ is an open subset of $C^{\infty}\left(\mathbb{R}^{n}, \mathbb{R}\right)$.

Proof. We now define the following mapping

$$
\Psi_{a}: C^{\infty}\left(\mathbb{R}^{n}, \mathbb{R}\right) \rightarrow C^{\infty}\left(\mathbb{R} \times \mathbb{R}^{n}, \mathbb{R}^{n}\right)
$$

by $\Psi_{a}(\phi)=F[a, \phi]$. If we consider a mapping

$$
a_{*}: C^{\infty}\left(\mathbb{R}^{n}, \mathbb{R}\right) \rightarrow C^{\infty}\left(\mathbb{R}^{n}, \mathbb{R}^{n}\right)
$$

defined by $a_{*}(\phi)=a \circ \phi$, then if is continuous for Whitney $C^{\infty}$-topologies (cf., [6]). It follows that $\Phi_{a}$ is also continuous, so that the statement follows from the well-known fact (cf., [6]) that

$$
\tilde{T}_{S}=\left\{\Phi \in C^{\infty}\left(\mathbb{R} \times \mathbb{R}^{n}, \mathbb{R}^{n}\right) \mid j_{1}^{\ell} \Phi \text { is transversal to } \hat{S}\right\} .
$$

is open subset of $C^{\infty}\left(\mathbb{R} \times \mathbb{R}^{n}, \mathbb{R}^{n}\right)$ when Sisclosed.

We need the following fundamental transversality lemma (cf., [6]).

Lemina B.3. Let $X, B$ and $Z$ be smooth manifolds with $W$ a submanifold of $Z$. Let $j: B \rightarrow C^{\infty}(X, Y)$ be mapping (not necessarily continuous) and define $\Phi: X \times B \rightarrow Y$ by $\Phi(x, b)=j(b)(x)$. Assume that $\Phi$ is smooth and that $\Phi$ is transversal to $W$. Then the set $\{b \in B \mid j(b)$ is transversal to $W\}$ is dense in $B$.

There is the canonical identification on $\mathbb{R}$-vector spaces (cf., $[10,13]$ ):

$$
T_{z} J^{\ell}(n, n) \equiv\left(\mathfrak{M}_{u}\right)^{n} \bmod \mathfrak{M}_{u}^{\ell+1},
$$

where $\left(\mathfrak{M}_{u}\right)^{n}$ denotes the $n$-fold Cartesian product of $\mathfrak{M}_{u}$. For a map germ $f$ : $\left(\mathbb{R}^{n}, 0\right) \rightarrow\left(\mathbb{R}^{n}, 0\right)$, the tangent space of the orbit through $z=j_{0}^{\ell} f$ under the action of the $\mathcal{A}$-equivalence is given by

$$
T(\mathcal{A})(z)=\left\langle\frac{\partial f}{\partial u_{1}}, \ldots, \frac{\partial f}{\partial u_{n}}\right\rangle_{\mathfrak{M}_{u}}+\left(f^{*}\left(\mathfrak{M}_{u}\right)\right)^{n} \bmod \mathfrak{M}_{u}^{\ell+1}
$$

Then we have the following simple lemma. 
Lemma B.4. Let $f:\left(\mathbb{R}^{n}, 0\right) \rightarrow\left(\mathbb{R}^{n}, 0\right)$ be a map germ of the form $f\left(u_{1}, \ldots, u_{n}\right)=$ $\left(f_{1}\left(u_{1}, \ldots, u_{n}\right), u_{2}, \ldots, u_{n}\right)$. Then we have

$$
\left(\mathfrak{M}_{u}\right)^{n}=\mathfrak{M}_{u} \times\{0\}+T(\mathcal{A})\left(j_{0}^{\ell} f\right) \bmod \mathfrak{M}_{u}^{\ell+1} .
$$

Proof of Theorem $B .1$. Let $B$ be the space of polynomial functions of $\left(\mathbb{R}^{n}, 0\right) \rightarrow$ $(\mathbb{R}, 0)$ of degree at most $\ell$. Define a smooth mapping,

$$
g[a, \phi]: \mathbb{R} \times \mathbb{R}^{n} \times B^{\prime} \rightarrow \mathbb{R}^{n}
$$

by $g[a, \phi](t, u, b)=F[a, \phi+b](t, u)$. Then the Jacobian matrix of $g[a, \phi]$ with respect to $u$-variables at $u_{0}$ is given as follows:

$$
\left(\begin{array}{cccc}
1+c_{11} & c_{12} & \ldots & c_{1 n} \\
c_{21} & 1+c_{22} & \ldots & c_{2 n} \\
\vdots & \vdots & \ddots & \vdots \\
c_{n 1} & c_{n 2} & \ldots & 1+c_{n n}
\end{array}\right)
$$

where $c_{i j}=t \frac{d a_{i}}{d y}\left((\phi+b)\left(u_{0}\right)\right) \frac{\partial(\phi+b)}{\partial u_{n}}\left(u_{0}\right)$.

Define a mapping

$$
\Phi: \mathbb{R} \times \mathbb{R}^{n} \times B^{\prime} \rightarrow J^{\ell}\left(\mathbb{R}^{n}, \mathbb{R}^{n}\right)
$$

by

$$
\Phi(t, u, b)=j_{1}^{\ell} g[a, \phi](t, u, b)=j^{\ell} g[a, \phi]_{(t, b)}(u),
$$

where $g[a, \phi]_{(t, b)}(u)=g[a, \phi](t, b, u)$.

In order to apply Lemma B.3, we need to know that $\Phi$ is transversal to $\hat{S}$. Assuming that this transversality condition holds, then given $\phi: \mathbb{R}^{n} \rightarrow \mathbb{R}$ we can find a sequence $b_{1}, b_{2}, \ldots \in B$ converging to 0 so that $\lim _{k \rightarrow \infty}\left(\phi+b_{k}\right)=\phi$ in $C^{\infty}\left(\mathbb{R}^{n}, \mathbb{R}\right)$ and $T_{S}$ is dense in $C^{\infty}\left(\mathbb{R}^{n}, \mathbb{R}\right)$.

Without the loss of generality we may consider at the point $\left(t_{0}, 0,0\right) \in \mathbb{R} \times \mathbb{R}^{n} \times B$ and assume that $\phi(0)=0$. If $t_{0} \frac{d a_{i}}{d y}(0)=0$ for any $i=1, \ldots, n$, then $g[a, \phi]_{\left(t_{0}, 0\right)}$ is local diffeomorphism at the origin, so that the $\mathcal{A}$-orbit through $j_{0}^{\ell} g[a, \phi]_{\left(t_{0}, 0\right)}$ is open subset. Since $S$ is an $\mathcal{A}$-invariant submanifold, $\Phi$ is transversal to $\hat{S}$ at $\left(t_{0}, 0,0\right)$. We now assume that $t_{0} \frac{d a_{i}}{d y}(0) \neq 0$ for some $i=1, \ldots, n$. Then the mapping $a: \mathbb{R} \rightarrow \mathbb{R}^{n}$ is an immersion at 0 . It follows that there exist diffeomorphism germs $h:\left(\mathbb{R}^{n}, a(0)\right) \rightarrow\left(\mathbb{R}^{n}, 0\right)$ and $k:(\mathbb{R}, 0) \rightarrow(\mathbb{R}, 0)$ such that ho $a \circ k(y)=(y, 0, \ldots, 0)$. We consider a smooth mapping

$$
\tilde{g}[h \circ a \circ k, \phi]: \mathbb{R} \times \mathbb{R}^{n} \times B^{\prime} \rightarrow \mathbb{R}^{n}
$$

defined by

$$
\tilde{g}[h \circ a \circ k, \phi](t, u, b)=F[h \circ a \circ h, \phi+b](t, u)=\left(u_{1}+t(\phi+b)(u), u_{2}, \ldots, u_{n}\right) .
$$

By Lemma B.4 and since $S$ is $\mathcal{A}$-invariant, we can easily show that $\tilde{\Phi} \mid\left\{t_{0}\right\} \times\{0\} \times B$ is transversal to $S$ at $\left(t_{0}, 0,0\right)$, where $\tilde{\Phi}(t, u, b)=j_{1}^{\ell} \tilde{g}[h \circ a \circ k, \phi](t, u, b)$. 
On the other hand, we define $\mathbb{R}$-linear isomorphisms

$$
T h:\left(\mathfrak{M}_{u}\right)^{n} \rightarrow\left(\mathfrak{M}_{u}\right)^{n}
$$

by

and

$$
T h(\xi)=\left(\frac{\partial h_{i}}{\partial u_{j}}\right) \cdot \xi
$$

$$
t k: \mathfrak{M}_{u} \rightarrow \mathfrak{M}_{u}
$$

by

$$
t k(\eta)=\left(\frac{d k_{1}}{d y} \cdot \eta, \ldots, \frac{d k_{n}}{d y} \cdot \eta\right)
$$

Under the canonical identification, we can show that

$$
d \tilde{\Phi} \mid\left\{t_{0}\right\} \times\{0\} \times B=T h \circ\left(d \Phi \mid\left\{t_{0}\right\} \times\{0\} \times B\right) \circ t k \bmod \mathfrak{M}_{u}^{\ell+1} .
$$

Since $S$ is an $\mathcal{A}$-invariant submanifold, we have $T h(T S)=T S$, so that $\Phi \mid\left\{t_{0}\right\} \times$ $\{0\} \times B$ is transversal to $S$ at $\left(t_{0}, 0,0\right)$. Of course $\Phi$ is transversal to $\hat{S}$ at $\left(t_{0}, 0,0\right)$. This completes the proof.

Remark. We can also prove the multi-jet version of Theorem B.1. Thus the list of the classification of multi-germs in Section 7 is the generic list for initial data $\phi \in C^{\infty}\left(\mathbb{R}^{n}, \mathbb{R}\right)$. However the proof is rather a tedious, so that we omit it.

\section{REFERENCES}

1. V. I. ARNOL'D, Geometric Methods in the Theory of Ordinary Differential Equations, Springer-Verlag, 1983.

2. D. P. BALLOU, Solutions to nonlinear hyperbolic Cauchy problems without convexity conditions, Trans: Amer. Math. Soc. 152 (1970), 441-460.

3. N. M. CHEN, On types of singularities for solutions of nonlinear hyperbolic systems, Bull. Inst. Math. Acad. Sinica 10 (1982), 405-416.

4. J. DAMON, The unfolding and determinacy theorems for subgroups of $\mathcal{A}$ and $\mathcal{K}$, Memoirs of Amer. Math. Soc. 50-306 (1984).

5. J. GLIMM, D. MARCHESIN and O. MCBRYAN, Unstable fingers in two phase flows, Commun. Pure Appl. Math., 34 (1981), 53-75.

6. M. GOLUBITSKY and V. GUILLEMIN, Sable mappings and their singularities, Graduate Texts in Math. 14, Springer-Verlag, 1973.

7. J. GUCKENHEIMER, Solving a single conservation law, Lecture notes in Mathematics 468, Springer Verlag, New York, 1975, pp. 108-134.

8. G. JENNINGS, Piecewise smooth solutions of a single conservation law exist, Adv. in Math 33 (1979), 192-205.

9. S. N. KRUZKOV, First order quasilinear equations in several independent variables, Math. USSR Sb. 10 (1970), 217-243.

10. J. MARTINET, Singularities of Smooth Functions and Maps, London Mathematical Society Lecture Note Series 58, Cambridge university press, 1982.

11. S. NAKANE, Formation of shocks for a single conservation law, SIAM J. Math. Anal. 19 (1988), 1391-1408.

12. D. WAGNER, The Riemann problem in two space dimensions for a single conservation law, SIAM J. Math. Ann. 14 (1983), 534-559.

13. C. T. C. WALL, Finite determinacy of smooth map germs, Bull. London. Math. Soc. 14 (1981), 481-539.

14. G. WASSERMANN, Stability of unfoldings in space and time, Acta. Math. 135 (1975), 57128.

15. V. M. ZAKALYUKIN, Reconstructions of fronts and caustics depending on a parameter and versality of mappings, Itogi Nauki, Contemporary Problems in Mathematics 22 (1983), 53-93. 